Supplementary Information on the paper

\title{
Quantum-Chemical Study of the Effect of Triethylaluminum on the Chain-End Structure and Tacticity of Poly(N,N-Dimethylacrylamide) with Lithium Counterion in THF
}

by Alexander V. Yakimansky and Axel H. E. Müller*

Table 1_Suppl. Calculated total energies for aluminum hydride, dimethyl ether, and their complex.

\begin{tabular}{|c|c|c|c|}
\hline & \multicolumn{3}{|c|}{ Total energy, Hartree } \\
\hline Level of theory & $\mathbf{A l H}_{\mathbf{3}}$ & $\mathbf{H}_{\mathbf{3}} \mathbf{C}-\mathbf{O}-\mathbf{C H}_{\mathbf{3}}$ & $\mathbf{A l H}_{\mathbf{3}} \ldots \mathbf{H}_{\mathbf{3}} \mathbf{C}-\mathbf{O}-\mathbf{C H}_{\mathbf{3}}$ \\
\hline BP86/SVP//BP86/SVP & -244.13839 & -154.90085 & -399.07611 \\
\hline B3LYP/TZVP//BP86/SVP & -244.16663 & -154.98976 & -399.18896 \\
\hline MP2/TZVPP//BP86/SVP & -243.73346 & -154.72623 & -398.49931 \\
\hline
\end{tabular}


Table 2_Suppl. Total energies, $E$, and averaged energies per one DMPA-Li or $\mathrm{Et}_{3} \mathrm{Al}$ fragment, $\bar{E}$, calculated at the BP86/SVP//BP86/SVP and B3LYP/TZVP//BP86/SVP (marked bold) levels of theory, and ${ }^{13} \mathrm{C}$ NMR shifts for $\mathrm{C}^{\alpha}$-atoms for different complexes and/or aggregates of chain-end units, DMPA-Li.

\begin{tabular}{|c|c|c|c|}
\hline Structure & Fig. & E, Hartree & $\bar{E}$, Hartree \\
\hline THF & & $\begin{array}{l}-232.27070 \\
-232.38473\end{array}$ & - \\
\hline DMAA & & $\begin{array}{l}-325.66709 \\
-325.82370\end{array}$ & - \\
\hline iso-trimer & & $\begin{array}{l}-1017.58007 \\
\mathbf{- 1 0 1 8 . 0 4 8 3 7} \\
\end{array}$ & - \\
\hline hetero-trimer & & $\begin{array}{l}-1017.58013 \\
\mathbf{- 1 0 1 8 . 0 4 9 4 4}\end{array}$ & - \\
\hline syndio-trimer & & $\begin{array}{l}-1017.57670 \\
\mathbf{- 1 0 1 8 . 0 4 6 7 9} \\
\end{array}$ & - \\
\hline $\mathrm{Et}_{3} \mathrm{Al}$ & & $\begin{array}{l}-479.84532 \\
-\mathbf{4 7 9 . 9 8 2 5 7}\end{array}$ & $\begin{array}{l}-479.84532 \\
-479.98257\end{array}$ \\
\hline$\left(\mathrm{Et}_{3} \mathrm{Al}\right)_{2}$ & & $\begin{array}{l}-959.71075 \\
\mathbf{- 9 5 9 . 9 7 1 9 8}\end{array}$ & $\begin{array}{l}-479.85538 \\
-479.98599\end{array}$ \\
\hline $\mathrm{Et}_{3} \mathrm{Al} \bullet \mathrm{THF}$ & & $\begin{array}{l}-712.14519 \\
-712.39240\end{array}$ & $\begin{array}{l}-479.87449 \\
-480.00767\end{array}$ \\
\hline $\mathrm{Et}_{3} \mathrm{Al} \bullet \mathrm{DMAA}$ & & $\begin{array}{l}-805.54448 \\
\mathbf{- 8 0 5 . 8 3 3 7 2} \\
\end{array}$ & $\begin{array}{l}-479.87739 \\
-325.82605 \\
\end{array}$ \\
\hline $\begin{array}{c}(\mathrm{DMPA}-\mathrm{Li})_{2} \\
\bullet 4 \mathrm{THF}\end{array}$ & $1 \mathrm{a}$ & $\begin{array}{l}-1596.86886 \\
\mathbf{- 1 5 9 7 . 6 3 7 7 8} \\
\end{array}$ & $\begin{array}{l}-333.89304 \\
-334.04943 \\
\end{array}$ \\
\hline $\begin{array}{c}(\text { DMPA-Li) })_{1} \\
\bullet 3 T H F\end{array}$ & $1 \mathrm{~b}$ & $\begin{array}{l}-1030.69643 \\
\mathbf{- 1 0 3 1 . 1 9 4 5 2}\end{array}$ & $\begin{array}{l}-333.88434 \\
-334.04033\end{array}$ \\
\hline $\begin{array}{l}(\mathrm{DMPA}-\mathrm{Li})_{1} \\
\text {-3THF•Et }{ }_{3} \mathrm{Al}\end{array}$ & 1c & $\begin{array}{l}-1510.58764 \\
\mathbf{- 1 5 1 1 . 2 1 7 9 3}\end{array}$ & $\begin{array}{l}-333.90106 \\
-334.05607\end{array}$ \\
\hline iso-trimer $\bullet \mathrm{Et}_{3} \mathrm{Al}$ & $2 \mathrm{a}$ & $\begin{array}{l}-1497.45092 \\
\mathbf{- 1 4 9 8 . 0 5 3 0 7}\end{array}$ & $\begin{array}{l}-479.87085 \\
-\mathbf{4 8 0 . 0 0 4 7 0}\end{array}$ \\
\hline hetero-trimer $\bullet \mathrm{Et}_{3} \mathrm{Al}$ & $2 b$ & $\begin{array}{l}-1497.45590 \\
\mathbf{- 1 4 9 8 . 0 5 7 5 4}\end{array}$ & $\begin{array}{l}-479.87577 \\
-\mathbf{4 8 0 . 0 0 8 1 0}\end{array}$ \\
\hline syndio-trimer $\bullet \mathrm{Et}_{3} \mathrm{Al}$ & 2c & $\begin{array}{l}-1497.45660 \\
\mathbf{- 1 4 9 8 . 0 5 7 9 7}\end{array}$ & $\begin{array}{l}-479.87990 \\
-\mathbf{4 8 0 . 0 1 1 1 8}\end{array}$ \\
\hline
\end{tabular}


Table 3_Suppl. Total energies, $E$, and averaged energies per one triadic chain end fragment, $\bar{E}$, calculated at the BP86/SVP//BP86/SVP and B3LYP/TZVP//BP86/SVP (marked bold) levels of theory, for different triadic models of PDMAA-Li chain ends.

\begin{tabular}{|c|c|c|c|c|c|}
\hline Structure & Fig. & $\begin{array}{l}\text { Coordination } \\
\text { of PU to } \mathrm{Li}^{+}\end{array}$ & $\begin{array}{l}\text { Coordination } \\
\text { of APU to } \mathrm{Li}^{+}\end{array}$ & $E$, Hartree & $\bar{E}$, Hartree \\
\hline$(\sim m x)_{1} \bullet 3 \mathrm{THF}$ & $3 a$ & - & - & $\begin{array}{l}-1682.11295 \\
\mathbf{- 1 6 8 2 . 9 0 4 6 4}\end{array}$ & \begin{tabular}{|l}
-985.30086 \\
$\mathbf{- 9 8 5 . 7 5 0 4 4}$
\end{tabular} \\
\hline$(\sim r x)_{1} \bullet 3 \mathrm{THF}$ & $3 \mathrm{~b}$ & - & - & $\begin{array}{l}-1682.11069 \\
\mathbf{- 1 6 8 2 . 9 0 3 2 5} \\
\end{array}$ & \begin{tabular}{|l|}
-985.29857 \\
$\mathbf{- 9 8 5 . 7 4 9 0 5}$ \\
\end{tabular} \\
\hline$(\sim m x)_{1} \bullet 2 \mathrm{THF}$ & - & yes & - & $\begin{array}{l}-1449.83482 \\
\mathbf{- 1 4 5 0 . 5 1 4 2 4}\end{array}$ & \begin{tabular}{|l}
-985.29342 \\
$\mathbf{- 9 8 5 . 7 4 4 7 8}$ \\
\end{tabular} \\
\hline$(\sim r x)_{1} \bullet 2 \mathrm{THF}$ & - & yes & - & $\begin{array}{r}-1449.83482 \\
\mathbf{- 1 4 5 0 . 5 1 3 1 8} \\
\end{array}$ & \begin{tabular}{|l|}
-985.29342 \\
$\mathbf{- 9 8 5 . 7 4 3 7 2}$ \\
\end{tabular} \\
\hline$(\sim m x)_{1} \bullet 2 \mathrm{THF}$ & - & - & yes & $\begin{array}{l}-1449.83281 \\
-\mathbf{1 4 5 0 . 5 1 0 9 4} \\
\end{array}$ & \begin{tabular}{|l|}
-985.29141 \\
$\mathbf{9 8 5 . 7 4 1 4 8}$ \\
\end{tabular} \\
\hline$(\sim r x)_{1} \bullet 2 \mathrm{THF}$ & - & - & yes & $\begin{array}{l}-1449.82942 \\
\mathbf{- 1 4 5 0 . 5 0 8 7 2} \\
\end{array}$ & \begin{tabular}{|l|}
-985.28803 \\
$\mathbf{- 9 8 5 . 7 3 9 2 6}$ \\
\end{tabular} \\
\hline$(\sim m x)_{1} \bullet \mathrm{THF}$ & - & yes & yes & $\begin{array}{c}-1217.54769 \\
-\end{array}$ & $\begin{array}{c}-985.27699 \\
- \\
\end{array}$ \\
\hline$(\sim r x)_{1} \bullet \mathrm{THF}$ & - & yes & yes & $\begin{array}{c}-1217.54853 \\
- \\
\end{array}$ & $\begin{array}{c}-985.27783 \\
- \\
\end{array}$ \\
\hline$(\sim m x)_{2} \bullet 4 \mathrm{THF}$ & $4 a$ & - & - & $\begin{array}{l}-2899.67543 \\
\mathbf{- 2 9 0 1 . 0 3 2 3 6} \\
\end{array}$ & \begin{tabular}{|l|}
-985.29633 \\
$\mathbf{9 8 5 . 7 4 6 6 7}$ \\
\end{tabular} \\
\hline$(\sim r x)_{2} \bullet 4 \mathrm{THF}$ & $4 b$ & - & - & $\begin{array}{l}-2899.68087 \\
\mathbf{- 2 9 0 1 . 0 3 8 8 1}\end{array}$ & \begin{tabular}{|l|}
-985.29904 \\
$\mathbf{- 9 8 5 . 7 4 9 9 4}$ \\
\end{tabular} \\
\hline $\begin{array}{c}(m x)_{1} \bullet(r x)_{1} \\
\bullet 4 \mathrm{THF}\end{array}$ & - & - & - & $\begin{array}{c}-2899.67832 \\
-\end{array}$ & $\begin{array}{c}-985.29777 \\
-\end{array}$ \\
\hline $\begin{array}{c}(\sim m m)_{1} \bullet 3 \mathrm{THF} \\
\bullet \mathrm{Et}_{3} \mathrm{Al} \\
\end{array}$ & $5 a$ & - & - & $\begin{array}{l}-2161.99226 \\
-\mathbf{2 1 6 2 . 9 1 6 9 1} \\
\end{array}$ & \begin{tabular}{|l}
-985.30568 \\
$\mathbf{- 9 8 5 . 7 5 5 0 4}$ \\
\end{tabular} \\
\hline $\begin{array}{c}(\sim m r)_{1} \bullet 3 \mathrm{THF} \\
\bullet \mathrm{Et}_{3} \mathrm{Al}\end{array}$ & $5 b$ & - & - & $\begin{array}{l}-2161.99791 \\
-\mathbf{2 1 6 2 . 9 2 2 7 1} \\
\end{array}$ & \begin{tabular}{|l|}
-985.31133 \\
$\mathbf{- 9 8 5 . 7 6 0 8 5}$ \\
\end{tabular} \\
\hline $\begin{array}{c}(\sim r m)_{1} \bullet 3 \mathrm{THF} \\
\bullet \mathrm{Et}_{3} \mathrm{Al}\end{array}$ & $5 c$ & - & - & $\begin{array}{l}-2161.99290 \\
-2162.91678 \\
\end{array}$ & \begin{tabular}{|l}
-985.30632 \\
$-\mathbf{9 8 5 . 7 5 4 9 2}$ \\
\end{tabular} \\
\hline $\begin{array}{c}(\sim r r)_{1} \bullet 3 \mathrm{THF} \\
\bullet \mathrm{Et}_{3} \mathrm{Al}\end{array}$ & $5 d$ & - & - & $\begin{array}{l}-2161.99647 \\
-\mathbf{2 1 6 2 . 9 2 0 2 7}\end{array}$ & \begin{tabular}{|l|}
-985.30989 \\
$\mathbf{- 9 8 5 . 7 5 8 4 0}$ \\
\end{tabular} \\
\hline
\end{tabular}




\section{Structure 1a}

forcefield $\mathrm{mm}+$

sys $0 \quad 0 \quad 1$

view $40 \quad 0.1304555150 .9981181 \quad 0.002907359-0.06125126 \quad-0.00455098 \quad 0.9996328$ $\begin{array}{llllllll}0.0267117 & 0.06115111 & 0.02694018 & 0.9977649 & -1.9057 & 0.16614 & -55.084\end{array}$

seed -1111

mol 1

atom 1 - Li ** $-01.0056970 .7828294-0.0415595642$ s 15 s 16 s 42 s atom $2-0 * *-02.9319890 .78785690 .125645531 \mathrm{~s} 3$ a $41 \mathrm{~s}$

atom $3-C^{* *}-03.8093981 .7668180 .156266832$ a 4 s 6 d

atom $4-N^{* *}-03.5549482 .7554031 .17552633 \mathrm{~s} 5 \mathrm{~s} 85 \mathrm{~s}$

atom $5-C^{* *}-03.2294612 .2378032 .4930544 \mathrm{~s} 9 \mathrm{~s} 10 \mathrm{~s} 11 \mathrm{~s}$

atom $6-C^{* *}-04.8811061 .909097-0.707457633$ d 7 s 8 s

atom $7-C^{* *}-05.1236151 .013511-1.89394946 \mathrm{~s} 12 \mathrm{~s} 13 \mathrm{~s} 14 \mathrm{~s}$

atom $8-H^{* *}-05.6050722 .722653-0.546521116 \mathrm{~s}$

atom $9-\mathrm{H}^{* *}-02.6078022 .9661573 .06407115 \mathrm{~s}$

atom $10-\mathrm{H}^{* *}-04.1463492 .019253 .11118515 \mathrm{~s}$

atom $11-H^{* *}-02.6615351 .2942382 .36980615 \mathrm{~s}$

atom $12-\mathrm{H}^{* *}-06.1572890 .5878193-1.90462117 \mathrm{~s}$

atom $13-\mathrm{H}^{* *}-05.0062621 .539998-2.87656117 \mathrm{~s}$

atom $14-\mathrm{H}^{* *}-04.4183830 .154428-1.89849617 \mathrm{~s}$

atom $15-0 * *-00.64491312 .216943-1.46451431 \mathrm{~s} 17 \mathrm{~s} 18 \mathrm{~s}$

atom $16-0 * *-0-0.57151441 .412011 .14097731 \mathrm{~s} 19 \mathrm{~s} 20 \mathrm{~s}$

atom $17-C^{* *}-01.3582292 .138963-2.720068415$ s 22 s 25 s $26 \mathrm{~s}$

atom $18-C_{* *}-00.68142083 .573836-0.9557078415$ s 21 s 27 s $28 \mathrm{~s}$

atom $19-C^{* *}-0-0.66351771 .5297292 .571949416$ s 24 s 29 s 30 s

atom $20-C_{* *}-0-1.8605591 .0404480 .5819569416$ s 23 s 31 s $32 \mathrm{~s}$

atom $21-C^{* *}-01.1591744 .438911-2.127966418 \mathrm{~s} 22 \mathrm{~s} 33 \mathrm{~s} 34 \mathrm{~s}$

atom $22-C^{* *}-02.1041373 .47086-2.862038417 \mathrm{~s} 21 \mathrm{~s} 35 \mathrm{~s} 36 \mathrm{~s}$

atom $23-C^{* *}-0-2.8681071 .2066341 .726113420$ s 24 s 37 s $38 \mathrm{~s}$

atom $24-C^{* *}-\odot-2.0024210 .88955612 .959063419$ s 23 s 39 s 40 s

atom $25-\mathrm{H}^{* *}-02.040211 .263235-2.67953117 \mathrm{~s}$

atom $26-\mathrm{H}^{* *}-00.62050311 .984362-3.543613117 \mathrm{~s}$

atom $27-H^{* *}-0-0.33484483 .83666-0.5912412118 \mathrm{~s}$

atom $28-\mathrm{H}^{* *}-\odot 1.3967963 .613029-0.1005032118 \mathrm{~s}$

atom $29-\mathrm{H}^{* *}-00.21556291 .0246473 .027165119 \mathrm{~s}$

atom $30-\mathrm{H}^{* *}-0-0.62897132 .6096152 .857198119 \mathrm{~s}$

atom $31-\mathrm{H}^{* *}-0-2.0617191 .700131-0.2890417120 \mathrm{~s}$

atom $32-H^{* *}-0-1.80331-0.014431090 .2239194120 \mathrm{~s}$

atom $33-H^{* *}-00.30381124 .725327-2.780953121 \mathrm{~s}$

atom $34-H^{* *}-01.6607045 .370772-1.790433121 \mathrm{~s}$

atom $35-\mathrm{H}^{* *}-03.0812663 .408865-2.333144122 \mathrm{~s}$

atom $36-\mathrm{H}^{* *}-\odot 2.2865943 .748681-3.922107 \quad 122 \mathrm{~s}$

atom $37-H^{* *}-0-3.2456952 .2526461 .775243123 \mathrm{~s}$

atom $38-H^{* *}-0-3.7430630 .53112441 .620167123 \mathrm{~s}$

atom $39-H^{* *}-0-1.884489-0.21077873 .074249124 \mathrm{~s}$

atom $40-\mathrm{H}^{* *}-\Theta-2.4141991 .2954873 .907564124 \mathrm{~s}$

atom 41 - Li ** $-02.849362-1.1231870 .0392747942$ s 42 s 55 s 56 s

atom $42-0 * *-00.918735-1.129814-0.0666883131$ s 41 s 43 a

atom $43-C^{* *}-00.04933453-2.105835-0.2105237342$ a 44 s 46 d

atom $44-N^{* *}-00.1614667-3.146795 \quad 0.7818572343 \mathrm{~s} 45 \mathrm{~s} 81 \mathrm{~s}$

atom $45-C_{* *}-00.2964554-2.6992632 .157074444$ s 49 s 50 s $51 \mathrm{~s}$

atom $46-C^{* *}-0-0.8896592-2.199316-1.22264343$ d $47 \mathrm{~s} 48 \mathrm{~s}$

atom $47-C^{* *}-0-0.9661076-1.242644-2.383484446$ s 52 s 53 s $54 \mathrm{~s}$

atom $48-\mathrm{H}^{* *}-0-1.626086-3.017467-1.207483146 \mathrm{~s}$

atom $49-\mathrm{H}^{* *}-00.8342549-3.4584722 .771406145 \mathrm{~s}$

atom $50-\mathrm{H}^{* *}-\odot-0.6990809-2.5096342 .650538145 \mathrm{~s}$

atom $51-H^{* *}-00.872611-1.752672 .163955145 \mathrm{~s}$

atom $52-\mathrm{H}^{* *}-0-1.989634-0.8131868-2.51745147 \mathrm{~s}$

atom $53-\mathrm{H}^{* *}-0-0.7097387-1.717999-3.365731147 \mathrm{~s}$

atom $54-\mathrm{H}^{* *}-0-0.2703886-0.3870674-2.244298147 \mathrm{~s}$ 


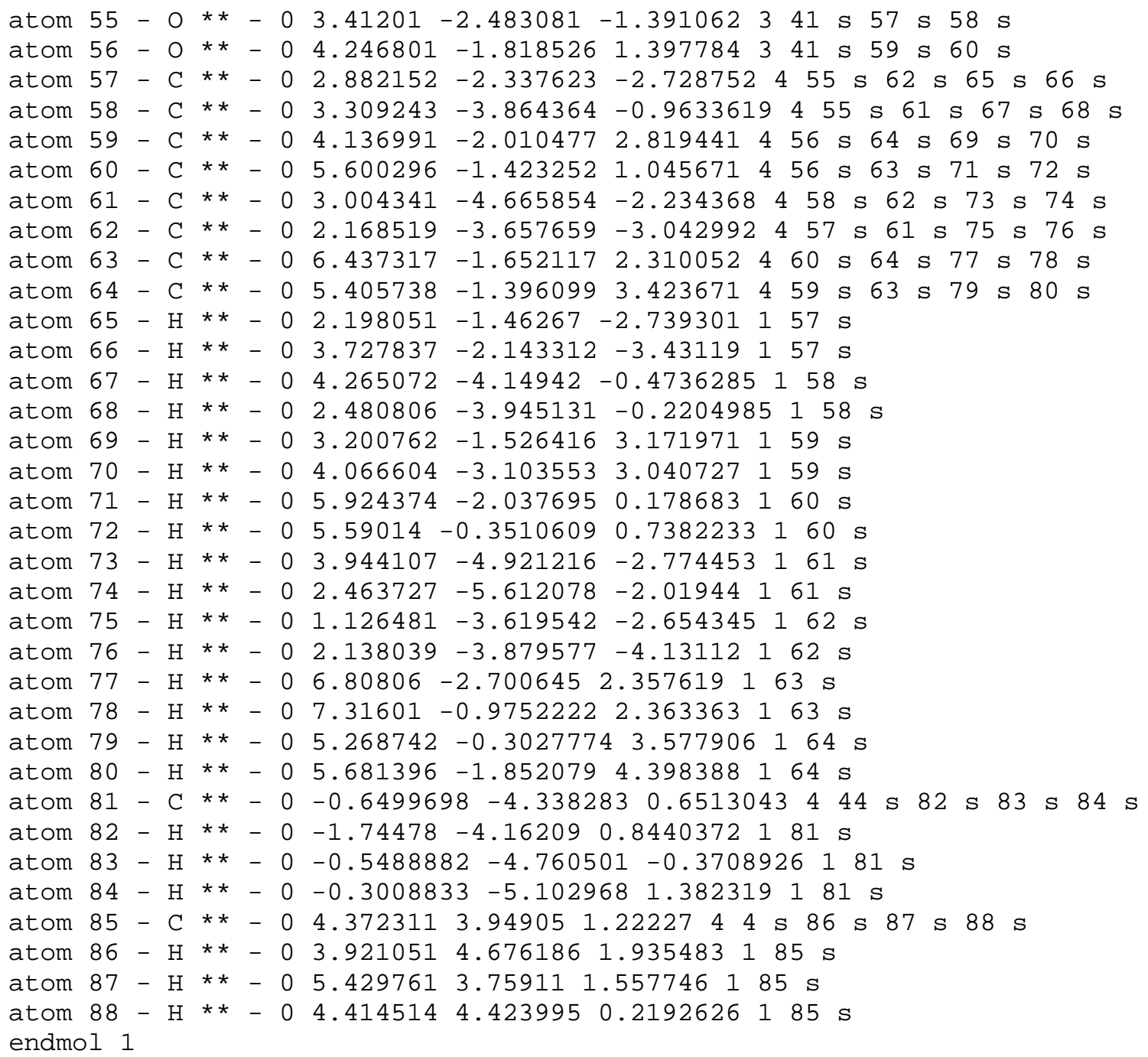

\section{Structure 1b}

forcefield $\mathrm{mm}+$

sys $\odot \quad \odot \quad 1$

view $40 \quad 0.1748955150 .9981181 \quad 0.002907359 \quad-0.06125126-0.00455098 \quad 0.9996328 \quad-$ $\begin{array}{llllllll}0.0267117 & 0.06115111 & 0.02694018 & 0.9977649 & -12.519 & -0.31412 & -54.547\end{array}$

seed -1111

$\operatorname{mol} 1$

atom 1 - Li ** - $\odot 11.26392-0.83504750 .123933342 \mathrm{~s} 15 \mathrm{~s} 16 \mathrm{~s} 41 \mathrm{~s}$

atom $2-0 * *-013.07247-0.9092355-0.243674221$ s 3 a

atom $3-C^{* *}-013.97047-0.001428246-0.52996932$ a 4 a $5 \mathrm{~s}$

atom $4-C_{* *}^{*} \odot 13.953090 .8302794-1.63967433$ a 7 s $8 \mathrm{~s}$

atom $5-\mathrm{N} * *-015.016350 .13568750 .460060733 \mathrm{~s} 6 \mathrm{~s} 54 \mathrm{~s}$

atom $6-\mathrm{C} * *-015.60127-1.1055490 .937296245 \mathrm{~s} 9 \mathrm{~s} 10 \mathrm{~s} 11 \mathrm{~s}$

atom $7-C^{* *}-012.850410 .8000555-2.66319844 \mathrm{~s} 12 \mathrm{~s} 13 \mathrm{~s} 14 \mathrm{~s}$

atom $8-\mathrm{H}^{* *}-014.817791 .47591-1.85869214 \mathrm{~s}$

atom $9-\mathrm{H}^{* *}-016.00913-0.98144281 .96831616 \mathrm{~s}$

atom $10-\mathrm{H}^{* *}-016.44121-1.4699340 .281713916 \mathrm{~s}$

atom $11-\mathrm{H}^{* *}-014.81312-1.8832980 .949937316 \mathrm{~s}$

atom $12-\mathrm{H}^{* *}-\odot 13.161550 .3431648-3.63998417 \mathrm{~s}$

atom $13-H^{* *}-012.459851 .817473-2.92216817 \mathrm{~s}$

atom $14-\mathrm{H}^{* *}-\odot 11.993020 .1939318-2.29341717 \mathrm{~s}$

atom $15-0 * *-010.85119-1.9076351 .79518931 \mathrm{~s} 17 \mathrm{~s} 18 \mathrm{~s}$

atom $16-0 * *-010.29253-1.776864-1.40063931 \mathrm{~s} 19 \mathrm{~s} 20 \mathrm{~s}$

atom $17-C^{* *}-011.94763-2.6849242 .307954415$ s 23 s 25 s 26 s atom $18-C * *-\odot 10.02113-1.4918012 .896536415$ s 24 s 27 s 28 s 


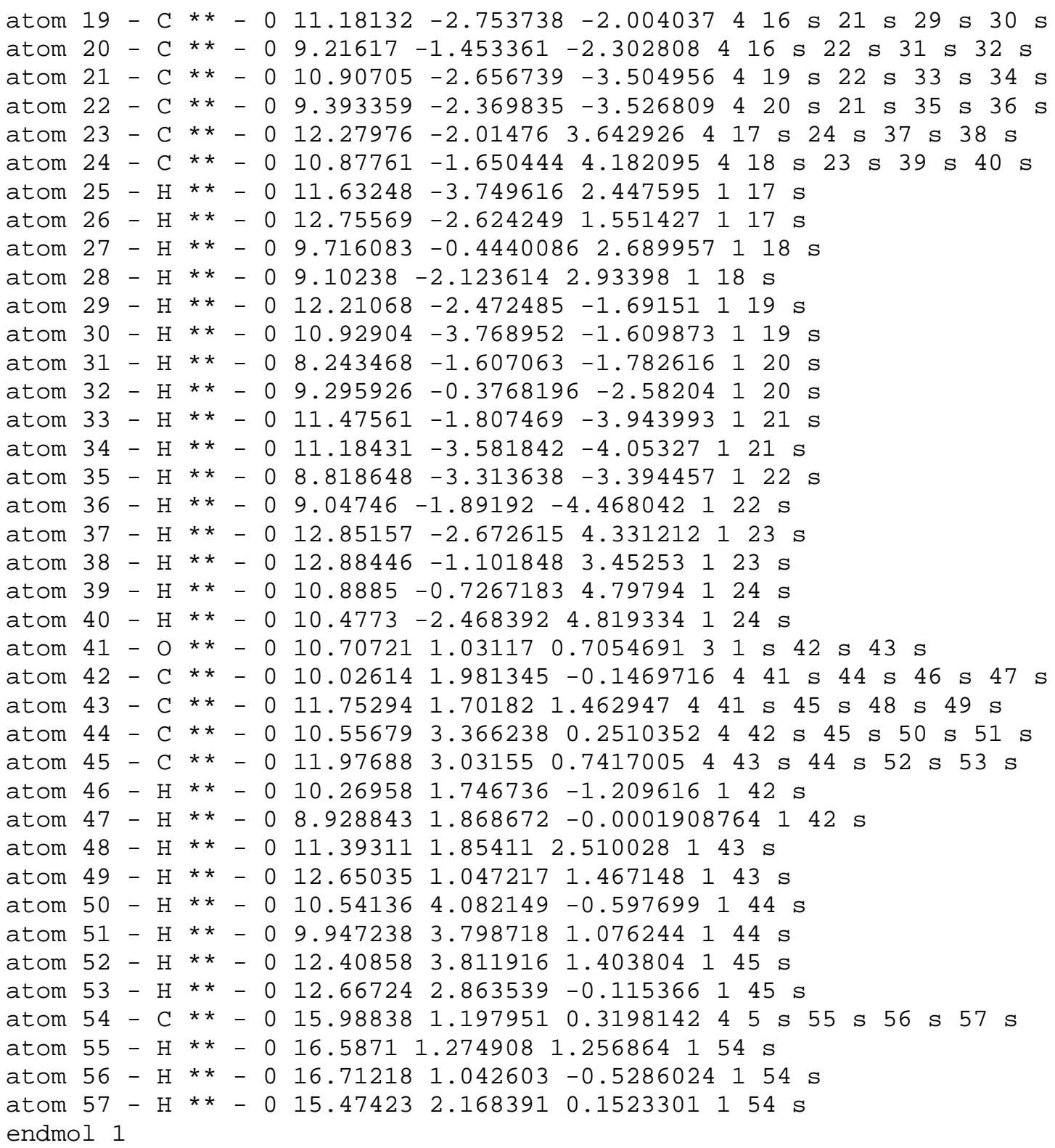

\section{Structure 1c}

forcefield $\mathrm{mm}+$

sys $\odot$

view $40 \quad 0.1399255150 .3285949 \quad-0.1922136 \quad-0.924705 \quad 0.09379404 \quad 0.9808732 \quad-$ $\begin{array}{llllllll}0.1705591 & 0.9398021 & -0.03068695 & 0.3403385 & -0.0062206 & -0.51711 & -55.32\end{array}$ $\operatorname{mol} 1$

atom 1 - C** -01.786480 .751232 .2854532 s 6 d 9 s

atom $2-C_{* *}-02.958950 .36421 .4253541$ s 3 s 4 s 5 s

atom $3-\mathrm{H}^{* *}-03.876510 .939151 .6970712 \mathrm{~s}$

atom $4-\mathrm{H}^{* *}-03.23611-0.717411 .5147712 \mathrm{~s}$

atom $5-\mathrm{H}^{* *}-02.747360 .569210 .3548412 \mathrm{~s}$

atom $6-C^{* *}-\odot 0.564191 .148161 .801131$ d 7 s $8 \mathrm{~s}$

atom $7-0 * *-00.219551 .028080 .4929236 \mathrm{~s} 18 \mathrm{~s} 58 \mathrm{~s}$

atom $8-\mathrm{N}^{* *}-0-0.426121 .743152 .6139736 \mathrm{~s} 10 \mathrm{~s} 14 \mathrm{~s}$

atom $9-\mathrm{H} * *-\odot 1.95490 .77973 .3738411 \mathrm{~s}$

atom $10-C^{* *}-\odot-0.092282 .128023 .9669848$ s 11 s 12 s $13 \mathrm{~s}$

atom $11-H^{* *}-00.874372 .673793 .97913110 \mathrm{~s}$

atom $12-H^{* *}-\odot-0.882962 .80614 .35861110 \mathrm{~s}$

atom $13-\mathrm{H}^{* *}-0-0.010451 .258434 .67736110 \mathrm{~s}$

atom $14-\mathrm{C} * *-\odot-1.813411 .360672 .4209748 \mathrm{~s} 15 \mathrm{~s} 16 \mathrm{~s} 17 \mathrm{~s}$ 
atom $15-\mathrm{H}^{* *}-0-1.9821 .123091 .35398114 \mathrm{~s}$ atom $16-\mathrm{H}^{* *}-\odot-2.107610 .468623 .04297114 \mathrm{~s}$ atom $17-\mathrm{H}^{* *}-\odot-2.487762 .202152 .69611114 \mathrm{~s}$ atom $18-\mathrm{Li} * *-0-0.00543-0.82415-0.0440347 \mathrm{~s} 20 \mathrm{~s} 19 \mathrm{~s} 45 \mathrm{~s}$ atom $19-0 * *-01.4605-1.96807-0.8918321$ s 22 s $18 \mathrm{~s}$ atom $20-0 * *-0-1.55635-1.19802-1.33217323 \mathrm{~s} 24 \mathrm{~s} 18 \mathrm{~s}$ atom $21-C * *-\odot 1.84722-3.27819-0.41922419 \mathrm{~s} 27 \mathrm{~s} 29 \mathrm{~s} 30 \mathrm{~s}$ atom $22-C^{* *}-\odot 2.37681-1.52158-1.92111419 \mathrm{~s} 28 \mathrm{~s} 31 \mathrm{~s} 32 \mathrm{~s}$ atom $23-C^{* *}-0-1.47391-2.1159-2.44475420$ s 25 s 33 s $34 \mathrm{~s}$ atom $24-C * *-0-2.67114-0.28529-1.51713420 \mathrm{~s} 26 \mathrm{~s} 35 \mathrm{~s} 36 \mathrm{~s}$ atom $25-C * *-0-2.7959-1.96531-3.20685423 \mathrm{~s} 26 \mathrm{~s} 37 \mathrm{~s} 38 \mathrm{~s}$ atom $26-C * *-0-3.12549-0.48112-2.96653424$ s 25 s 39 s 40 s atom $27-C * *-03.13793-3.6431-1.17235421$ s 28 s 41 s $42 \mathrm{~s}$ atom $28-C * *-03.02211-2.80101-2.45618422 \mathrm{~s} 27 \mathrm{~s} 43 \mathrm{~s} 44 \mathrm{~s}$ atom $29-\mathrm{H}^{* *}-01.98694-3.243150 .68297121 \mathrm{~s}$ atom $30-H^{* *}-01.01962-3.99149-0.64246121 \mathrm{~s}$ atom $31-H^{* *}-01.79545-0.94859-2.67179122 \mathrm{~s}$ atom $32-\mathrm{H}^{* *}-03.13383-0.83775-1.4716122 \mathrm{~s}$ atom $33-H^{* *}-0-0.60397-1.83653-3.08337123 \mathrm{~s}$ atom $34-H^{* *}-0-1.30355-3.13975-2.04555123 \mathrm{~s}$ atom $35-H^{* *}-\odot-3.47584-0.55699-0.79269124 \mathrm{~s}$ atom $36-H^{* *}-0-2.322090 .74467-1.29654124 \mathrm{~s}$ atom $37-H^{* *}-0-2.69823-2.22738-4.28156125 \mathrm{~s}$ atom $38-H^{* *}-\odot-3.58083-2.6185-2.76363125 \mathrm{~s}$ atom $39-H^{* *}-\odot-4.19841-0.23709-3.11412126 \mathrm{~s}$ atom $40-H^{* *}-0-2.524950 .16583-3.6422126 \mathrm{~s}$ atom $41-\mathrm{H}^{* *}-04.03026-3.32913-0.58764127 \mathrm{~s}$ atom $42-H^{* *}-03.22325-4.73347-1.36424127 \mathrm{~s}$ atom $43-H^{* *}-02.35396-3.29722-3.19509128 \mathrm{~s}$ atom $44-H^{* *}-03.99993-2.61392-2.94766128 \mathrm{~s}$ atom $45-0 * *-0-0.6267-2.090571 .47622346$ s 47 s $18 \mathrm{~s}$ atom $46-C * *-0-1.84811-2.859711 .38378445 \mathrm{~s} 48 \mathrm{~s} 50 \mathrm{~s} 51 \mathrm{~s}$ atom $47-C^{* *}-0-0.03958-2.25152 .78959445$ s 49 s 52 s $53 \mathrm{~s}$ atom $48-C * *-0-2.0841-3.456032 .78023446$ s 49 s 54 s $55 \mathrm{~s}$ atom $49-C^{* *}-0-0.65537-3.537083 .34611447 \mathrm{~s} 48 \mathrm{~s} 56 \mathrm{~s} 57 \mathrm{~s}$ atom $50-\mathrm{H}^{* *}-\odot-2.67227-2.195011 .0481146 \mathrm{~s}$ atom $51-H^{* *}-0-1.70911-3.654090 .6135146 \mathrm{~s}$ atom $52-\mathrm{H}^{* *}-01.06316-2.278342 .67258147 \mathrm{~s}$ atom $53-H^{* *}-0-0.28871-1.36693 .41823147 \mathrm{~s}$ atom $54-H^{* *}-0-2.70191-2.767543 .3979148 \mathrm{~s}$ atom $55-\mathrm{H}^{* *}-0-2.60323-4.436972 .74178148 \mathrm{~s}$ atom $56-H^{* *}-0-0.12523-4.432122 .94966149 \mathrm{~s}$ atom $57-\mathrm{H}^{* *}-0-0.62307-3.581374 .45524149 \mathrm{~s}$ atom $58-\mathrm{Al}$ ** $-00.136662 .55889-0.68347 \mathrm{~s} 59 \mathrm{~s} 66 \mathrm{~s} 73 \mathrm{~s}$ atom $59-C^{* *}-01.721973 .74886-0.29598458$ s 60 s 61 s $62 \mathrm{~s}$ atom $60-C * *-01.672724 .63480 .96403459 \mathrm{~s} 63 \mathrm{~s} 64 \mathrm{~s} 65 \mathrm{~s}$ atom $61-\mathrm{H}^{* *}-\odot 1.845074 .40639-1.19424159 \mathrm{~s}$ atom $62-H^{* *}-02.655493 .13311-0.27737159 \mathrm{~s}$ atom $63-\mathrm{H}^{* *}-02.561195 .306391 .07167160 \mathrm{~s}$ atom $64-\mathrm{H}^{* *}-00.772715 .292780 .96863160 \mathrm{~s}$ atom $65-\mathrm{H}^{* *}-01.620424 .021461 .89286160 \mathrm{~s}$ atom $66-C * *-0-1.645163 .49544-0.4046458$ s 67 s $68 \mathrm{~s} 69 \mathrm{~s}$ atom $67-\mathrm{C} * *-0-1.865614 .67733-1.37227466 \mathrm{~s} 70 \mathrm{~s} 71 \mathrm{~s} 72 \mathrm{~s}$ atom $68-\mathrm{H}^{* *}-0-2.516842 .7972-0.48839166 \mathrm{~s}$ atom $69-H^{* *}-0-1.686533 .873390 .64628166 \mathrm{~s}$ atom $70-H^{* *}-0-2.830845 .21927-1.20958167 \mathrm{~s}$ atom $71-\mathrm{H}^{* *}-0-1.056055 .43843-1.27871167 \mathrm{~s}$ atom $72-H^{* *}-0-1.862894 .34708-2.43812167 \mathrm{~s}$ atom $73-C^{* *}-\odot 0.249871 .67928-2.52768458$ s 74 s 75 s $76 \mathrm{~s}$ atom $74-C * *-01.544711 .93262-3.32608473$ s 77 s 78 s $79 \mathrm{~s}$ atom $75-H^{* *}-0-0.618952 .04182-3.13403173 \mathrm{~s}$ 
atom $76-\mathrm{H}^{* *}-\odot 0.081750 .57519-2.43497173 \mathrm{~s}$ atom $77-\mathrm{H}^{* *}-01.696243 .01973-3.51629174 \mathrm{~s}$ atom $78-\mathrm{H}^{* *}-02.450591 .59456-2.77032174 \mathrm{~s}$ atom $79-\mathrm{H}^{* *}-\odot 1.569681 .42891-4.32581174 \mathrm{~s}$ endmol 1

\section{Structure $\mathbf{2 a}$}

forcefield $\mathrm{mm}+$

sys $0 \circ 1$

view $40 \quad 0.148825515 \quad 0.9840676 \quad 0.1080037 \quad 0.1412309 \quad 0.1585171 \quad-0.1732284 \quad-$ $\begin{array}{llllllll}0.9720413 & -0.08051889 & 0.9789418 & -0.1875889 & -0.74193 & -2.472 & -55.511\end{array}$

seed -1111

mol 1

atom $1-C * *-\odot \odot \odot \odot 42$ s 5 s 6 s $7 \mathrm{~s}$

atom $2-C^{* *}-\odot 1.5547890041 \mathrm{~s} 3 \mathrm{~s} 4 \mathrm{~s} 39 \mathrm{~s}$

atom $3-C^{* *}-02.0631011 .464126042$ s 8 s 9 s $56 \mathrm{~s}$

atom $4-C^{* *}-\odot 2.056005-0.7450551 .23770432$ s 10 a $11 \mathrm{~d}$

atom $5-\mathrm{H}^{* *}-0-0.3389060 .633301-0.85041211 \mathrm{~s}$

atom $6-\mathrm{H}^{* *}-0-0.3410450 .5108730 .92753911 \mathrm{~s}$

atom $7-C * *-0-0.697822-1.380772-0.12288741$ s 43 s 44 s $45 \mathrm{~s}$

atom $8-\mathrm{H}^{* *}-01.6067471 .992968-0.86376713 \mathrm{~s}$

atom $9-\mathrm{H} * *-01.6720791 .9695660 .90942613 \mathrm{~s}$

atom $10-\mathrm{N} * *-\odot 2.776905-1.8992431 .15657734$ a $12 \mathrm{~s} 38 \mathrm{~s}$

atom $11-0 * *-01.762687-0.2868432 .37226324$ d $16 \mathrm{~s}$

atom $12-\mathrm{C} * *-\odot 3.181062-2.5892142 .384857410 \mathrm{~s} 13 \mathrm{~s} 14 \mathrm{~s} 15 \mathrm{~s}$

atom $13-\mathrm{H}^{* *}-04.270217-2.4487032 .570124112 \mathrm{~s}$

atom $14-H^{* *}-02.977365-3.6766752 .2766761112 \mathrm{~s}$

atom $15-\mathrm{H}^{* *}-02.615405-2.187673 .245556112 \mathrm{~s}$

atom 16 - Al ** -01.4095720 .6839734 .040799411 s 17 s $18 \mathrm{~s} 19 \mathrm{~s}$ atom $17-C^{* *}-01.051106-0.8687825 .283382416$ s 24 s 25 s $26 \mathrm{~s}$ atom $18-C * *-\odot-0.1433971 .8896153 .598258416$ s 20 s 21 s $34 \mathrm{~s}$ atom $19-\mathrm{C} * *-\odot 3.1589351 .6465994 .328801416 \mathrm{~s} 22 \mathrm{~s} 23 \mathrm{~s} 30 \mathrm{~s}$ atom $20-\mathrm{H}^{* *}-0-1.0423421 .2752043 .342751118 \mathrm{~s}$ atom $21-H^{* *}-\odot 0.09260832 .4524442 .660929118 \mathrm{~s}$ atom $22-\mathrm{H}^{* *}-\odot 3.0010332 .3331015 .198099119 \mathrm{~s}$ atom $23-H^{* *}-\odot 3.3396522 .3340283 .464545119 \mathrm{~s}$ atom $24-\mathrm{H}^{* *}-01.983981-1.4807215 .378615117 \mathrm{~s}$ atom $25-\mathrm{H}^{* *}-00.303365-1.5587314 .816204117 \mathrm{~s}$ atom $26-C^{* *}-00.563937-0.4927376 .69959417 \mathrm{~s} 27 \mathrm{~s} 28 \mathrm{~s} 29 \mathrm{~s}$ atom $27-\mathrm{H}^{* *}-00.400827-1.3789667 .360494126 \mathrm{~s}$ atom $28-\mathrm{H}^{* *}-01.2929580 .1663047 .225642126 \mathrm{~s}$ atom $29-\mathrm{H}^{* *}-\odot-0.4006620 .06412796 .668404126 \mathrm{~s}$ atom $30-C * *-\odot 4.412396 \odot .7902714 .588971419 \mathrm{~s} 31 \mathrm{~s} 32 \mathrm{~s} 33 \mathrm{~s}$ atom $31-\mathrm{H}^{* *}-04.2774950 .1223515 .470831130 \mathrm{~s}$ atom $32-H^{* *}-04.6485870 .1246573 .724354130 \mathrm{~s}$ atom $33-H^{* *}-\odot 5.3339731 .3924414 .786196130 \mathrm{~s}$ atom $34-C * *-\odot-0.5260052 .9056274 .697366418$ s 35 s 36 s $37 \mathrm{~s}$ atom $35-\mathrm{H}^{* *}-0-0.8326942 .3999075 .642099134 \mathrm{~s}$ atom $36-H^{* *}-00.3287323 .5717614 .957704134 \mathrm{~s}$ atom $37-H^{* *}-0-1.3721943 .5727394 .402281134 \mathrm{~s}$ atom $38-C * *-03.253687-2.543637-0.0627994410 \mathrm{~s} 40$ s 41 s 42 s atom $39-H^{* *}-01.898651-0.493775-0.9278512 \mathrm{~s}$ atom $40-H^{* *}-\odot 4.36176-2.646795-0.0235958138 \mathrm{~s}$ atom $41-H^{* *}-02.990647-1.963189-0.963407138 \mathrm{~s}$ atom $42-H^{* *}-02.814371-3.562316-0.156913138 \mathrm{~s}$ atom $43-C_{* *}-0-0.410622-2.086268-1.45970247$ s 70 s 71 s $72 \mathrm{~s}$ atom $44-C * *-0-2.223873-1.140974-0.017494237$ s 46 d $47 \mathrm{~s}$ atom $45-H^{* *}-0-0.353151-2.0391740 .70451617 \mathrm{~s}$ atom $46-0 * *-0-2.862844-0.84015-1.029515144 \mathrm{~d}$ atom $47-\mathrm{N} * *-0-2.822647-1.2413531 .225078344$ s 48 s $49 \mathrm{~s}$ atom $48-C * *-0-2.154013-1.6212562 .462216447$ s 50 s 51 s 52 s 


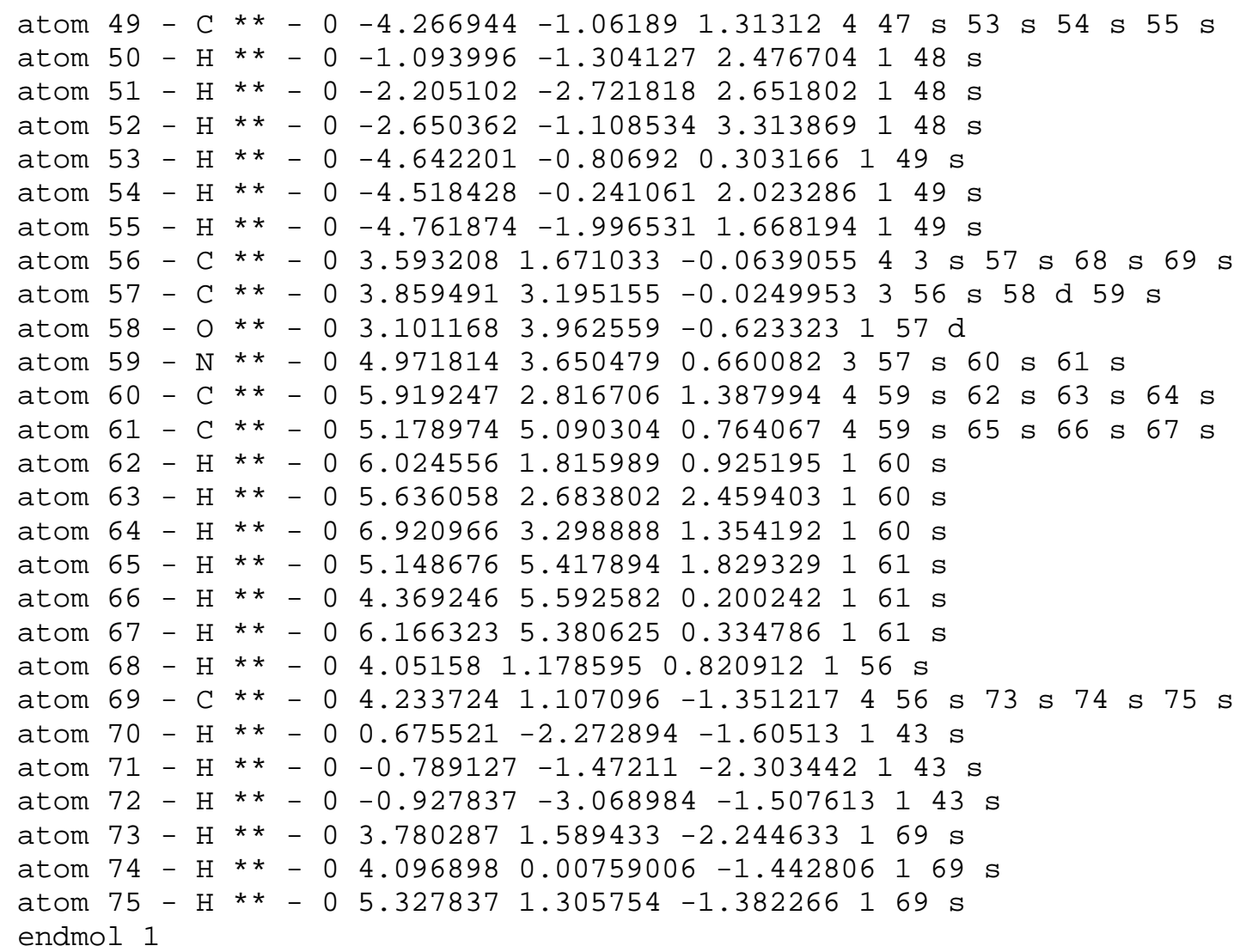

\section{Structure $2 b$}

forcefield $\mathrm{mm}+$

sys $\odot \odot 1$

$\begin{array}{llllllllll}\text { view } 40 & 0.15375 & 55 & 15 & 0.9808862 & -0.1156827 & -0.15646 & 0.1720948 & 0.1405224\end{array}$ $\begin{array}{lllllll}0.9750061 & -0.09080517 & -0.983296 & 0.1577449 & -0.86866 & 2.3304 & -55.909\end{array}$ 


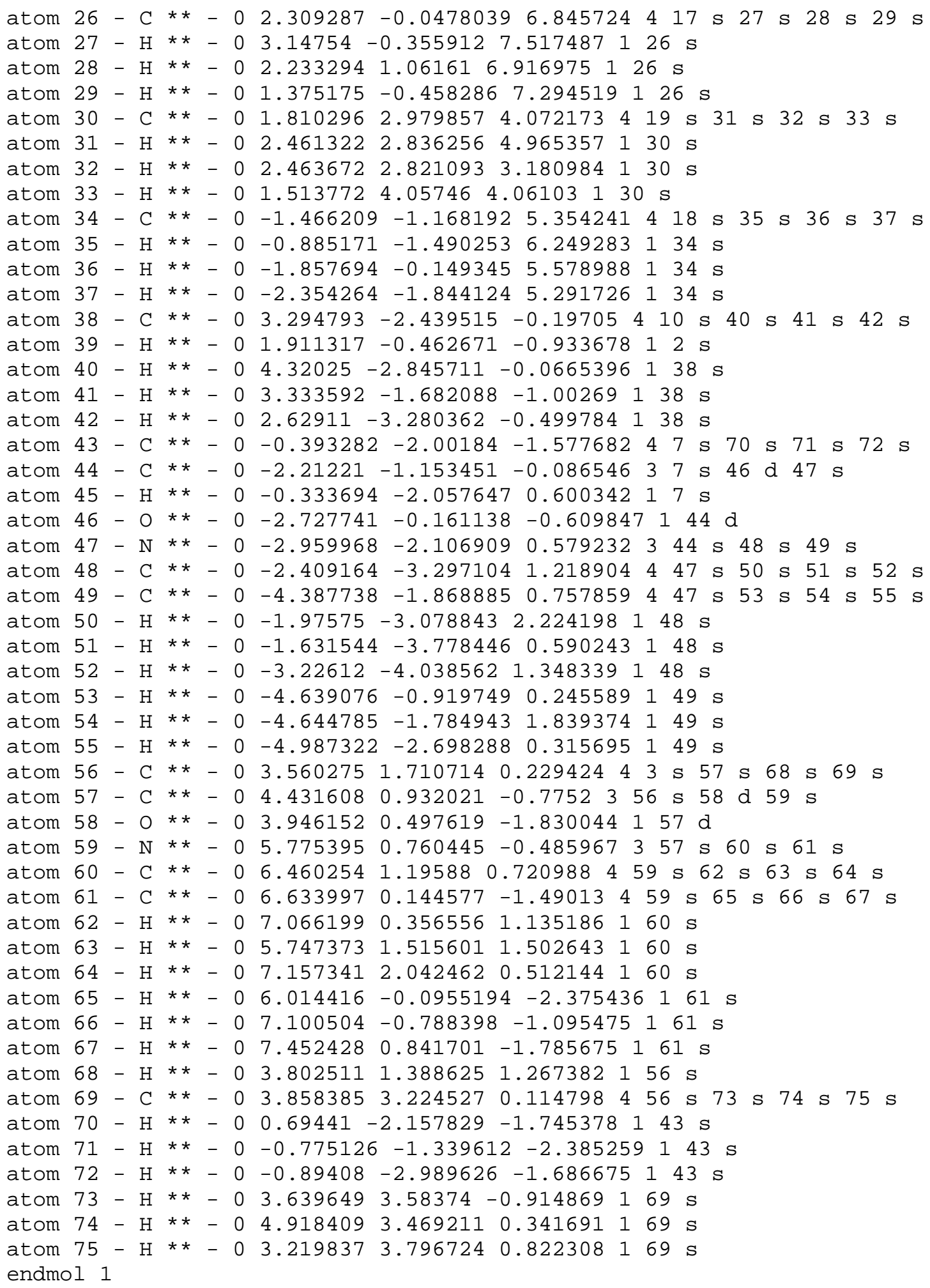

\section{Structure 2c}

forcefield $\mathrm{mm}+$

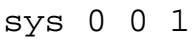

view $40 \quad 0.1423756 .3516 .35 \quad 0.9032616 \quad-0.372562 \quad 0.21287590 .41401110 .6263404 \quad-$ $\odot .66052440 .11275350 .6847593 \quad 0.719994-1.9822-0.95633-58.62$

seed -1111

$\operatorname{mol} 1$

atom $1-C * *-\odot \odot \odot \odot 42$ s 5 s 6 s $7 \mathrm{~s}$

atom $2-C^{* *}-\odot 1.554843 \odot \odot 41$ s 3 s 4 s $39 \mathrm{~s}$ 
atom $3-C^{* *}-02.0600821 .466748 \odot 42$ s 8 s 9 s 56 s

atom $4-C * *-02.130086-0.7466211 .19496432$ s 10 a $11 d$

atom $5-H^{* *}-\odot-0.3126570 .268088-1.03253311 \mathrm{~s}$

atom $6-H^{* *}-0-0.3589460 .8086730 .6733811 \mathrm{~s}$

atom $7-C^{* *}-0-0.73226-1.2883070 .42791841 \mathrm{~s} 43 \mathrm{~s} 44 \mathrm{~s} 45 \mathrm{~s}$

atom $8-\mathrm{H}^{* *}-01.5800081 .972712-0.8650313 \mathrm{~s}$

atom $9-\mathrm{H}^{* *}-\odot 1.7057391 .9672890 .92749913 \mathrm{~s}$

atom $10-\mathrm{N}^{* *}-02.956133-1.8117421 .00568634$ a $12 \mathrm{~s} 38 \mathrm{~s}$

atom $11-0 * *-01.866211-0.3642222 .36665324 \mathrm{~d} 16 \mathrm{~s}$

atom $12-\mathrm{C} * *-\odot 3.591966-2.4586482 .154953410 \mathrm{~s} 13 \mathrm{~s} 14 \mathrm{~s} 15 \mathrm{~s}$

atom $13-\mathrm{H}^{* *}-\odot 4.69877-2.3610842 .084541112 \mathrm{~s}$

atom $14-\mathrm{H}^{* *}-03.333717-3.5407242 .174295112 \mathrm{~s}$

atom $15-\mathrm{H} * *-\odot 3.240351-1.9822253 .088051112 \mathrm{~s}$

atom 16 - Al ** -01.2993950 .5099614 .010754411 s 17 s 18 s $19 \mathrm{~s}$ atom $17-C^{* *}-01.134937-1.1104065 .207859416 \mathrm{~s} 24 \mathrm{~s} 25 \mathrm{~s} 26 \mathrm{~s}$ atom $18-C * *-0-0.4127461 .4699283 .540271416$ s 20 s 21 s $34 \mathrm{~s}$ atom $19-\mathrm{C} * *-02.8623831 .7292424 .386789416 \mathrm{~s} 22 \mathrm{~s} 23 \mathrm{~s} 30 \mathrm{~s}$ atom $20-\mathrm{H}^{* *}-0-1.1603890 .7358573 .146245118 \mathrm{~s}$

atom $21-H^{* *}-0-0.2102622 .164872 .686993118 \mathrm{~s}$

atom $22-H^{* *}-\odot 2.6345272 .253915 .348348119 \mathrm{~s}$

atom $23-\mathrm{H}^{* *}-\odot 2.8811242 .5479843 .624162119 \mathrm{~s}$

atom $24-\mathrm{H}^{* *}-02.105764-1.6681795 .189067117 \mathrm{~s}$

atom $25-H^{* *}-\odot 0.390633-1.81874 .761623117 \mathrm{~s}$

atom $26-\mathrm{C} * *-00.751288-0.8394216 .678162417$ s 27 s 28 s $29 \mathrm{~s}$ atom $27-\mathrm{H}^{* *}-00.694482-1.7662987 .300369126 \mathrm{~s}$

atom $28-H^{* *}-01.485902-0.1656237 .177282126 \mathrm{~s}$

atom $29-\mathrm{H} * *-0-0.241336-0.3396546 .759437126 \mathrm{~s}$

atom $30-\mathrm{C} * *-\odot 4.2595211 .0876524 .487385419 \mathrm{~s} 31 \mathrm{~s} 32 \mathrm{~s} 33 \mathrm{~s}$

atom $31-\mathrm{H} * *-\odot 4.2928540 .2923695 .268183130 \mathrm{~s}$

atom $32-H^{* *}-04.5603020 .6005883 .52866130 \mathrm{~s}$

atom $33-\mathrm{H}^{* *}-05.0684261 .8167124 .739789130 \mathrm{~s}$

atom $34-C * *-\odot-1.0605042 .2746874 .688152418 \mathrm{~s} 35 \mathrm{~s} 36 \mathrm{~s} 37 \mathrm{~s}$

atom $35-\mathrm{H}^{* *}-0-1.3419931 .6198945 .544814134 \mathrm{~s}$

atom $36-H^{* *}-\odot-0.3652453 .0458935 .094038134 \mathrm{~s}$

atom $37-H_{* *}-0-1.9896342 .8139694 .380025134 \mathrm{~s}$

atom $38-C * *-03.3339-2.33808-0.314009410 \mathrm{~s} 40 \mathrm{~s} 41 \mathrm{~s} 42 \mathrm{~s}$

atom $39-H^{* *}-01.886862-0.474386-0.93881912 \mathrm{~s}$

atom $40-H^{* *}-04.144923-1.731625-0.776908138 \mathrm{~s}$

atom $41-H^{* *}-02.460817-2.376578-0.996926138 \mathrm{~s}$

atom $42-\mathrm{H}^{* *}-03.705828-3.37383-0.175068138 \mathrm{~s}$

atom $43-\mathrm{H}^{* *}-0-0.564034-1.4369681 .51602617 \mathrm{~s}$

atom $44-C^{* *}-\odot-0.251874-2.524817-0.34755537$ s 46 d $47 \mathrm{~s}$

atom $45-C * *-0-2.252743-1.1056370 .20170647$ s 73 s 74 s 75 s

atom $46-0 * *-00.237023-2.417047-1.482287144 \mathrm{~d}$

atom $47-\mathrm{N} * *-0-0.42492-3.7578110 .255272344$ s 48 s $49 \mathrm{~s}$

atom $48-C * *-0-0.922005-3.9408291 .618164447$ s 50 s 51 s 52 s

atom $49-\mathrm{C} * *-0-0.108669-4.960328-0.507581447$ s 53 s 54 s 55 s

atom $50-\mathrm{H}^{* *}-0-1.977216-3.6068791 .73946148 \mathrm{~s}$

atom $51-\mathrm{H}^{* *}-0-0.30064-3.4049452 .369725148 \mathrm{~s}$

atom $52-\mathrm{H} * *-0-0.88419-5.0211171 .863935148 \mathrm{~s}$

atom $53-\mathrm{H}^{* *}-00.219961-4.644884-1.51693149 \mathrm{~s}$

atom $54-\mathrm{H}^{* *}-0-1.003519-5.618137-0.600791149 \mathrm{~s}$

atom $55-\mathrm{H}^{* *}-00.706254-5.546179-0.0213598149 \mathrm{~s}$

atom $56-C * *-03.5829611 .671017-0.11209343$ s 57 s 68 s $69 \mathrm{~s}$

atom $57-\mathrm{C} * *-04.1010141 .156467-1.469321356 \mathrm{~s} 58$ d $59 \mathrm{~s}$

atom $58-0 * *-03.3918031 .223347-2.477409157 \mathrm{~d}$

atom $59-\mathrm{N} * *-05.3966350 .658536-1.516858357 \mathrm{~s} 60 \mathrm{~s} 61 \mathrm{~s}$

atom $60-\mathrm{C} * *-06.2975370 .616864-0.367421459 \mathrm{~s} 62$ s $63 \mathrm{~s} 64 \mathrm{~s}$

atom $61-\mathrm{C} * *-05.9501590 .290326-2.816395459 \mathrm{~s} 65$ s 66 s $67 \mathrm{~s}$

atom $62-\mathrm{H}^{* *}-05.8975-0.005130450 .463926160 \mathrm{~s}$

atom $63-\mathrm{H}^{* *}-06.5251471 .6289480 .0388632160 \mathrm{~s}$ 
atom $64-\mathrm{H}^{* *}-07.2571990 .162608-0.688288160 \mathrm{~s}$ atom $65-\mathrm{H}^{* *}-06.783730 .971398-3.108569161 \mathrm{~s}$ atom $66-\mathrm{H}^{* *}-05.1374120 .374024-3.564282161 \mathrm{~s}$ atom $67-\mathrm{H}^{* *}-06.336843-0.75431-2.802832161 \mathrm{~s}$ atom $68-C^{* *}-03.9217343 .1757320 .00902514456$ s 70 s 71 s 72 s atom $69-H^{* *}-04.082291 .1424020 .728554156 \mathrm{~s}$ atom $70-H^{* *}-03.4308943 .745895-0.809674168 \mathrm{~s}$ atom $71-H^{* *}-05.0157193 .365108-0.0535891168 \mathrm{~s}$ atom $72-H^{* *}-03.562623 .5773860 .981745168 \mathrm{~s}$ atom $73-H^{* *}-0-2.465942-0.96258-0.8802771445 \mathrm{~s}$ atom $74-\mathrm{H}^{* *}-0-2.616715-0.2095390 .750254145 \mathrm{~s}$ atom $75-H^{* *}-0-2.843057-1.979820 .551726145 \mathrm{~s}$ endmol 1

\section{Structure 3a}

forcefield $\mathrm{mm}+$

sys $\odot$ ○ 1

$\begin{array}{lllllllllllllllll}\text { view } 4 \odot & 0.1262255 & 15 & 1 & \odot & \odot & \odot & 1 & \odot & \odot & \odot & 1 & -2.2832 & 12.83 & -52.8\end{array}$

seed -1111

$\operatorname{mol} 1$

atom $1-C * *-0-0.2479112-15.04296-1.73216432 \mathrm{~s} 10$ a $16 \mathrm{~s}$ atom $2-C * *-\odot 1.187355-15.0627-2.17766241$ s 3 s 4 s $5 \mathrm{~s}$ atom $3-C^{* *}-02.303618-14.85521-1.08726942 \mathrm{~s} 6 \mathrm{~s} 7 \mathrm{~s} 13 \mathrm{~s}$ atom $4-\mathrm{H}^{* *}-01.429815-16.01438-2.70621712 \mathrm{~s}$ atom $5-H^{* *}-\odot 1.346354-14.23526-2.90998112 \mathrm{~s}$ atom $6-C_{* *}-03.602125-14.41617-1.79032543$ s 8 s 9 s $29 \mathrm{~s}$ atom $7-H^{* *}-01.959115-14.03948-0.420738213 \mathrm{~s}$ atom $8-\mathrm{H}^{* *}-03.863942-15.20119-2.53366216 \mathrm{~s}$ atom $9-\mathrm{H}^{* *}-\odot 3.37493-13.48961-2.36672916 \mathrm{~s}$ atom $10-C * *-0-0.9435047-13.84365-1.65865431$ a 11 a $12 \mathrm{~s}$ atom $11-0 * *-0-0.4015423-12.66571-1.79279210$ a $46 \mathrm{~s}$ atom $12-\mathrm{N} * *-0-2.372884-13.82869-1.461619310 \mathrm{~s} 17 \mathrm{~s} 21 \mathrm{~s}$ atom $13-C_{* *}-02.560504-16.15293-0.309529933 \mathrm{~s} 14 \mathrm{~d} 15 \mathrm{~s}$ atom $14-0 * *-03.387272-16.97959-0.7322668113 \mathrm{~d}$ atom $15-N_{* *}-01.864572-16.383810 .8625834313$ s 25 s 42 s atom $16-\mathrm{H}^{* *}-\odot-0.7453407-16.00209-1.52780811 \mathrm{~s}$

atom $17-C_{* *}-0-3.088861-15.08379-1.384699412 \mathrm{~s} 18 \mathrm{~s} 19 \mathrm{~s} 20 \mathrm{~s}$ atom $18-\mathrm{H}^{* *}-\odot-2.891429-15.66109-0.4390999117 \mathrm{~s}$ atom $19-\mathrm{H}^{* *}-0-4.184379-14.88744-1.433752117 \mathrm{~s}$ atom $20-H^{* *}-\odot-2.81179-15.73305-2.2423131117 \mathrm{~s}$ atom $21-\mathrm{C} * *-\odot-2.887218-12.80258-0.5722483412$ s 22 s 23 s 24 s atom $22-\mathrm{H}^{* *}-0-2.26469-11.89482-0.6985301121 \mathrm{~s}$ atom $23-H^{* *}-0-3.946956-12.56116-0.8233005121 \mathrm{~s}$ atom $24-H^{* *}-0-2.858156-13.108290 .5116315121 \mathrm{~s}$ atom $25-\mathrm{C} * *-02.181293-17.572331 .6401415 \mathrm{~s} 26 \mathrm{~s} 27 \mathrm{~s} 28 \mathrm{~s}$ atom $26-H^{* *}-02.527872-17.294182 .664176125 \mathrm{~s}$ atom $27-\mathrm{H}^{* *}-\odot 1.285973-18.22961 .746274125 \mathrm{~s}$ atom $28-H^{* *}-02.982711-18.124251 .111537125 \mathrm{~s}$ atom $29-C_{* *}-04.840159-14.13434-0.899055746 \mathrm{~s} 30 \mathrm{~s} 31 \mathrm{~s} 86 \mathrm{~s}$ atom $30-\mathrm{H}^{* *}-05.050314-15.04398-0.2996718129 \mathrm{~s}$ atom $31-C * *-06.023683-13.8413-1.838524329 \mathrm{~s} 32$ d $33 \mathrm{~s}$ atom $32-0 * *-\odot 6.272566-12.68067-2.195187131 \mathrm{~d}$ atom $33-\mathrm{N} * *-06.756279-14.91669-2.312007331 \mathrm{~s} 34 \mathrm{~s} 38 \mathrm{~s}$ atom $34-C_{* *}-07.894562-14.65372-3.178775433$ s 35 s 36 s $37 \mathrm{~s}$ atom $35-\mathrm{H}^{* *}-07.971032-13.55817-3.32252134 \mathrm{~s}$ atom $36-H^{* *}-08.83712-15.03804-2.72143134 \mathrm{~s}$ atom $37-H^{* *}-07.765575-15.15179-4.169031134 \mathrm{~s}$ atom $38-C * *-06.528186-16.31928-1.984699433$ s $39 \mathrm{~s} 40 \mathrm{~s} 41 \mathrm{~s}$ atom $39-\mathrm{H}^{* *}-\odot 6.604927-16.92747-2.915188138 \mathrm{~s}$ atom $40-H^{* *}-\odot 7.299148-16.70148-1.271235138 \mathrm{~s}$ atom $41-H^{* *}-\odot 5.520791-16.49733-1.557456138 \mathrm{~s}$ 
atom $42-C_{* *}-00.8634512-15.495221 .436335415$ s 43 s 44 s 45 s atom $43-H^{* *}-00.0518229-16.107971 .89002142 \mathrm{~s}$ atom $44-H^{* *}-01.291311-14.852172 .246116142 \mathrm{~s}$ atom $45-H^{* *}-00.3938885-14.872940 .6468384142 \mathrm{~s}$ atom $46-\mathrm{Li} * *-00.3930438-11.38986-2.845891411 \mathrm{~s} 47$ s 48 s $73 \mathrm{~s}$ atom $47-0 * *-02.01103-11.49154-4.075784346$ s 49 s $50 \mathrm{~s}$ atom $48-0 * *-01.103971-9.981503-1.544847346$ s 51 s $52 \mathrm{~s}$ atom $49-C * *-\odot 1.97777-12.21374-5.326444447$ s 55 s 57 s 58 s atom $50-C * *-03.345201-10.99057-3.818915447$ s 56 s $59 \mathrm{~s} 60 \mathrm{~s}$ atom 51 - C** - $\odot 1.089402-8.54107-1.563446448$ s 53 s 61 s $62 \mathrm{~s}$ atom 52 - C** - $\odot 1.159509-10.46386-0.1754578448$ s 54 s 63 s 64 s atom $53-C * *-01.172778-8.088058-0.09334135451$ s 54 s 65 s 66 s atom $54-C_{* *}-00.611599-9.3079680 .6626074452$ s 53 s 67 s 68 s atom $55-C^{* *}-03.288365-11.865-6.040712449 \mathrm{~s} 56 \mathrm{~s} 69 \mathrm{~s} 70 \mathrm{~s}$ atom $56-C^{* *}-04.248573-11.67194-4.853436450$ s 55 s 71 s 72 s atom $57-\mathrm{H}^{* *}-01.905756-13.30699-5.114395149 \mathrm{~s}$ atom $58-H^{* *}-01.064741-11.90295-5.87819149 \mathrm{~s}$ atom $59-\mathrm{H}^{* *}-03.340268-9.880254-3.938469150 \mathrm{~s}$ atom $60-\mathrm{H}^{* *}-03.63084-11.23467-2.774873150 \mathrm{~s}$ atom $61-\mathrm{H}^{* *}-\odot 1.941457-8.172783-2.179139151 \mathrm{~s}$ atom $62-H^{* *}-00.142573-8.200938-2.045189151 \mathrm{~s}$ atom $63-H^{* *}-00.5657603-11.40243-0.1499492152 \mathrm{~s}$ atom $64-H^{* *}-02.218867-10.692690 .09191155152 \mathrm{~s}$ atom $65-H^{* *}-02.230798-7.9096450 .1993954153 \mathrm{~s}$ atom $66-H^{* *}-00.6063484-7.1514750 .09453106153 \mathrm{~s}$ atom $67-H^{* *}-0-0.5008343-9.3072550 .6430671154 \mathrm{~s}$ atom $68-\mathrm{H}^{* *}-\odot 0.9382635-9.3518371 .723306154 \mathrm{~s}$ atom $69-\mathrm{H}^{* *}-03.613045-12.65932-6.745655155 \mathrm{~s}$ atom $70-\mathrm{H} * *-03.179115-10.91731-6.61344155 \mathrm{~s}$ atom $71-\mathrm{H}^{* *}-\odot 5.145478-11.06598-5.095873156 \mathrm{~s}$ atom $72-\mathrm{H}^{* *}-04.603478-12.65122-4.463817156 \mathrm{~s}$ atom $73-0 * *-0 \quad-1.020489-10.86129-4.238358346$ s 74 s 75 s atom $74-C * *-\odot-1.296172-9.589514-4.857224473$ s 76 s 78 s 79 s atom $75-C_{* *}-0-2.138296-11.76714-4.432687473$ s 77 s 80 s 81 s atom $76-C * *-0-2.659209-9.735748-5.557192474$ s 77 s 82 s 83 s atom $77-C_{* *}-0-3.328088-10.85483-4.737111475$ s 76 s 84 s 85 s atom $78-\mathrm{H}^{* *}-0-1.326394-8.805069-4.064172174 \mathrm{~s}$ atom $79-\mathrm{H}^{* *}-0-0.4713-9.33524-5.560457174 \mathrm{~s}$ atom $80-H^{* *}-\odot-1.918218-12.44132-5.296206175 \mathrm{~s}$ atom $81-H * *-0-2.243364-12.38155-3.512141175 \mathrm{~s}$ atom $82-\mathrm{H}^{* *}-0-3.235364-8.786139-5.567997176 \mathrm{~s}$ atom $83-\mathrm{H}^{* *}-0-2.52323-10.06106-6.612112176 \mathrm{~s}$ atom $84-\mathrm{H}^{* *}-0-4.140165-11.37558-5.287502177 \mathrm{~s}$ atom $85-H^{* *}-0-3.756707-10.4485-3.793654177 \mathrm{~s}$ atom $86-C^{* *}-04.637773-12.958270 .06752706429 \mathrm{~s} 87$ s 88 s $89 \mathrm{~s}$ atom $87-H^{* *}-03.77341-13.144110 .7414548186 \mathrm{~s}$ atom $88-H^{* *}-04.462678-12.01435-0.4931744186 \mathrm{~s}$ atom $89-H^{* *}-\odot 5.537399-12.802380 .7022085186 \mathrm{~s}$ endmol 1

\section{Structure $3 b$}

forcefield $\mathrm{mm}+$

sys $\odot \quad 0 \quad 1$

$\begin{array}{lllllllllllllllll}\text { view } 4 \odot & 0.12919 & 55 & 15 & 1 & \odot & \odot & \odot & 1 & \odot & \odot & \odot & 1 & -15.485 & 12.876 & -52.518\end{array}$ seed -1111

$\operatorname{mol} 1$

atom 1 - C** - $014.07152-14.78357-2.81027532$ s 10 a $16 \mathrm{~s}$ atom $2-C * *-\odot 15.49737-14.60316-3.25279941$ s 3 s 4 s 5 s atom $3-C^{* *}-016.62111-14.5893-2.15151342 \mathrm{~s} 6 \mathrm{~s} 7 \mathrm{~s} 13 \mathrm{~s}$ atom $4-\mathrm{H}^{* *}-015.7821-15.39922-3.98153212 \mathrm{~s}$ atom $5-\mathrm{H}^{* *}-\odot 15.59601-13.62392-3.77880312 \mathrm{~s}$ 


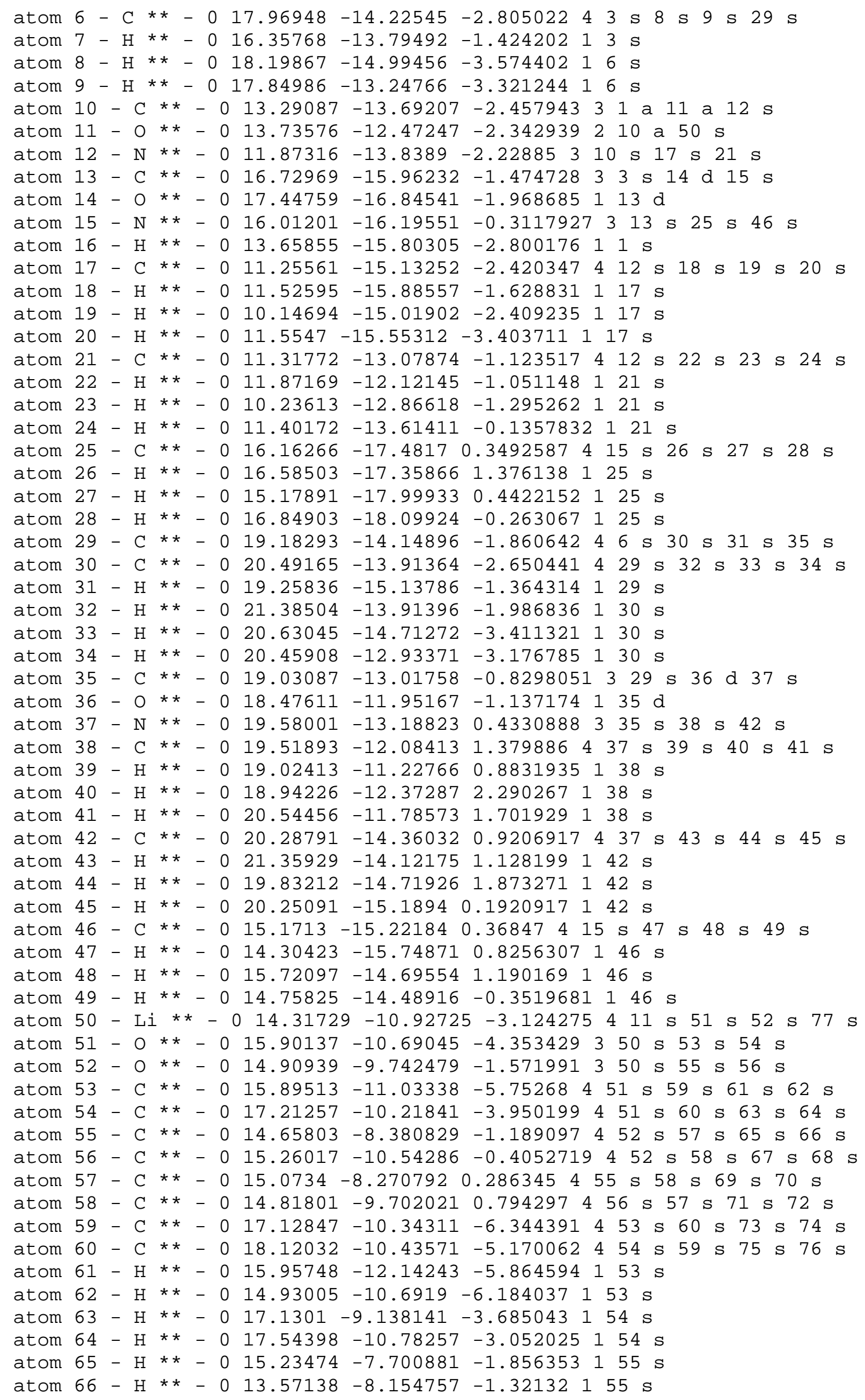




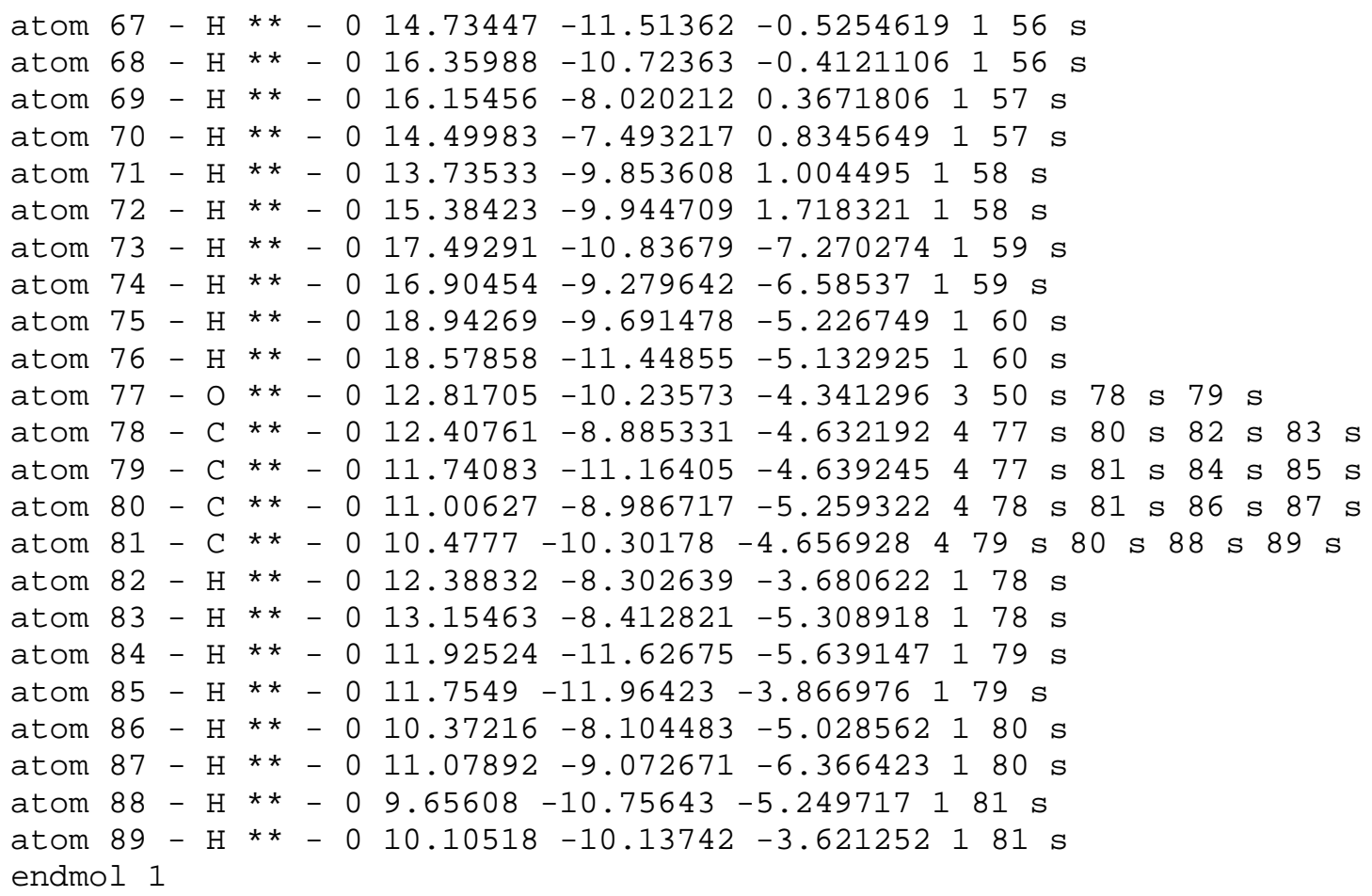

\section{Structure $4 a$}

forcefield $\mathrm{mm}+$

sys $\odot \circ 1$

$\begin{array}{llllllllll}\text { view } 40 & 0.12025 & 64.9 & 24.9 & 0.5597448 & -0.03829838 & -0.8277796 & -0.2191238 & 0.9565337\end{array}$ $\begin{array}{llllllll}-0.1924269 & 0.7991687 & 0.2890962 & 0.5270226 & -2.4545 & 3.385 & -64.364\end{array}$

seed -1111

mol 1

atom $1-0 * *-\odot \odot \odot \odot 32 \mathrm{~s} 5 \mathrm{~s} 14 \mathrm{~s}$

atom $2-C^{* *}-01.449414 \odot \odot 4 \quad 1$ s 3 s $12 \mathrm{~s} 13 \mathrm{~s}$

atom $3-C^{* *}-\odot 1.8712161 .475265 \odot 42 \mathrm{~s} 4 \mathrm{~s} 10 \mathrm{~s} 11 \mathrm{~s}$

atom $4-C^{* *}-00.6934482 .144005-0.73238243 \mathrm{~s} 5 \mathrm{~s} 8 \mathrm{~s} 9 \mathrm{~s}$

atom $5-C^{* *}-0-0.4926471 .344184-0.18572941$ s 4 s 6 s $7 \mathrm{~s}$

atom $6-\mathrm{H}^{* *}-0-1.3614261 .299473-0.876215 \mathrm{~s}$

atom $7-\mathrm{H}^{* *}-0-0.8401181 .7465950 .79629915 \mathrm{~s}$

atom $8-H^{* *}-00.7812292 .004519-1.83297614 \mathrm{~s}$

atom $9-\mathrm{H}^{* *}-00.605273 .23134-0.52078214 \mathrm{~s}$

atom $10-H^{* *}-01.9503691 .8503451 .04448613 \mathrm{~s}$

atom $11-\mathrm{H}^{* *}-02.848311 .636235-0.50307413 \mathrm{~s}$

atom $12-H^{* *}-01.796111-0.5582870 .89441112 \mathrm{~s}$

atom $13-\mathrm{H}^{* *}-01.800788-0.526454-0.91787712 \mathrm{~s}$

atom $14-\mathrm{Li} * *-0-1.056645-1.3935041 .10341441 \mathrm{~s} 15 \mathrm{~s} 28 \mathrm{~s} 104 \mathrm{~s}$

atom $15-0 * *-0-2.927282-0.657792 \quad 0.773408 \quad 3 \quad 14$ s 16 s 19 s

atom $16-C^{* *}-0-3.5851510 .406591 .470454415$ s 17 s 26 s 27 s

atom $17-C^{* *}-0-4.9618-0.1663541 .8785634416$ s 18 s 24 s 25 s

atom $18-C^{* *}-0-5.189699-1.3298580 .866917417$ s 19 s 22 s 23 s

atom $19-C^{* *}-0-3.939809-1.279676-0.0366834415$ s 18 s 20 s 21 s

atom $20-\mathrm{H}^{* *}-0-4.123548-0.656404-0.94395119 \mathrm{~s}$

atom $21-\mathrm{H}^{* *}-0-3.556662-2.266051-0.36529119 \mathrm{~s}$

atom $22-\mathrm{H}^{* *}-0-6.123428-1.2113290 .278276118 \mathrm{~s}$

atom $23-\mathrm{H}^{* *}-0-5.248932-2.3048871 .394387118 \mathrm{~s}$

atom $24-\mathrm{H}^{* *}-0-4.933921-0.5466092 .920826117 \mathrm{~s}$

atom $25-\mathrm{H}^{* *}-\odot \quad-5.755696 \quad 0.6085341 .826691117 \mathrm{~s}$

atom $26-\mathrm{H}^{* *}-0-2.928090 .7016562 .314423116 \mathrm{~s}$

atom $27-\mathrm{H}^{* *}-0-3.708517 \quad 1.2804820 .782918116 \mathrm{~s}$

atom $28-0^{* *}-0-1.039049-3.27598 \quad 0.782753314 \mathrm{~s} 29$ a $77 \mathrm{~s}$

atom $29-C^{* *}-0-1.4274-4.212007-0.0511476328$ a $30 \mathrm{~s} 39 \mathrm{~d}$ 
atom $30-N^{* *}-\odot-2.088511-5.3296020 .581802329 \mathrm{~s} 31 \mathrm{~s} 35 \mathrm{~s}$ atom $31-C * *-0-3.186233-4.9594181 .465498430 \mathrm{~s} 32 \mathrm{~s} 33 \mathrm{~s} 34 \mathrm{~s}$ atom $32-H^{* *}-\odot-3.409149-5.7841132 .181395131 \mathrm{~s}$ atom $33-H^{* *}-0-4.13596-4.7386920 .90225131 \mathrm{~s}$ atom $34-H^{* *}-\odot-2.897825-4.0530122 .035003131 \mathrm{~s}$ atom $35-C * *-\odot-2.387734-6.500054-0.219659430 \mathrm{~s} 36 \mathrm{~s} 37 \mathrm{~s} 38 \mathrm{~s}$ atom $36-H^{* *}-0-2.724688-7.3254870 .448225135 \mathrm{~s}$ atom $37-H^{* *}-0-3.196949-6.329727-0.981902135 \mathrm{~s}$ atom $38-\mathrm{H}^{* *}-0-1.475172-6.833944-0.757301135 \mathrm{~s}$ atom $39-C * *-0-1.223809-4.195412-1.419467329$ d 40 s 76 s atom $40-C * *-\odot-0.472359-3.095821-2.117219439$ s 41 s 74 s 75 s atom $41-C * *-\odot-1.345528-2.106178-2.956055440$ s 42 s 53 s 73 s atom $42-C * *-\odot-2.441176-2.761863-3.82434341$ s 43 d $44 \mathrm{~s}$ atom $43-0 * *-0-3.628784-2.481651-3.606115142 \mathrm{~d}$ atom $44-N * *-0-2.111552-3.646885-4.84347342$ s 45 s $49 \mathrm{~s}$ atom $45-C_{* *}-0-3.18956-4.302008-5.570044444 \mathrm{~s} 46$ s $47 \mathrm{~s} 48 \mathrm{~s}$ atom $46-\mathrm{H}^{* *}-0-3.104593-4.106363-6.665186145 \mathrm{~s}$ atom $47-H^{* *}-0-3.16063-5.406572-5.412396145 \mathrm{~s}$ atom $48-H^{* *}-\odot-4.14854-3.899041-5.190734145 \mathrm{~s}$ atom $49-C^{* *}-0-0.776788-4.083959-5.209986444$ s 50 s 51 s 52 s atom $50-H^{* *}-0-0.659168-4.047211-6.318618149 \mathrm{~s}$ atom $51-H^{* *}-0-0.00449071-3.433937-4.764317149 \mathrm{~s}$ atom $52-\mathrm{H}^{* *}-0-0.581894-5.134651-4.883223149 \mathrm{~s}$ atom $53-C * *-\odot-0.467064-1.045761-3.664117441$ s 54 s 71 s 72 s atom $54-C * *-0-1.189905-0.0647239-4.61465453$ s 55 s 66 s 70 s atom $55-C * *-0-0.1829611 .005077-5.085784354$ s 56 d $57 \mathrm{~s}$ atom $56-0 * *-00.6844041 .429191-4.312588155 \mathrm{~d}$ atom $57-N^{* *}-0-0.2908751 .507409-6.375187355$ s 58 s $62 \mathrm{~s}$ atom $58-C^{* *}-00.6474712 .536498-6.801548457$ s 59 s 60 s 61 s atom $59-\mathrm{H} * *-\odot 1.3651132 .710692-5.976791158 \mathrm{~s}$ atom $6 \odot-H^{* *}-\odot 1.1966652 .212129-7.716575158 \mathrm{~s}$ atom $61-\mathrm{H}^{* *}-00.1146163 .488403-7.037003158 \mathrm{~s}$ atom $62-C^{* *}-0-1.2974721 .158774-7.362637457$ s 63 s 64 s 65 s atom $63-H^{* *}-\odot-2.0192640 .421341-6.967826162 \mathrm{~s}$ atom $64-H^{* *}-0-1.8695732 .06531-7.673882162 \mathrm{~s}$ atom $65-H_{* *}-0-0.8249180 .729825-8.27929162 \mathrm{~s}$ atom $66-C * *-\odot-2.3975970 .648245-3.96274454$ s 67 s 68 s $69 \mathrm{~s}$ atom $67-\mathrm{H}^{* *}-0-2.0680871 .215007-3.06411166 \mathrm{~s}$ atom $68-H^{* *}-0-2.8659941 .373461-4.665673166 \mathrm{~s}$ atom $69-H^{* *}-0-3.169721-0.0911064-3.661172166 \mathrm{~s}$ atom $70-\mathrm{H}^{* *}-\odot-1.565028-0.653906-5.479442154 \mathrm{~s}$ atom $71-\mathrm{H}^{* *}-00.0347062-0.45256-2.86954153 \mathrm{~s}$ atom $72-H^{* *}-00.36076-1.531867-4.231558153 \mathrm{~s}$ atom $73-H^{* *}-0-1.964013-1.563917-2.213213141 \mathrm{~s}$ atom $74-\mathrm{H}^{* *}-00.340856-3.507184-2.765011140 \mathrm{~s}$ atom $75-H^{* *}-\odot 0.0373864-2.467754-1.3535140 \mathrm{~s}$ atom $76-\mathrm{H}^{* *}-\odot-1.660783-4.984434-2.050108139 \mathrm{~s}$ atom $77-\mathrm{Li} * *-00.100818-3.433882 .35037428 \mathrm{~s} 78 \mathrm{~s} 91 \mathrm{~s} 104 \mathrm{~s}$ atom $78-0 * *-01.862212-4.3003751 .700327377$ s 79 s $82 \mathrm{~s}$ atom $79-C^{* *}-02.451157-3.9191760 .432008478$ s 80 s 89 s $90 \mathrm{~s}$ atom $80-C * *-03.010547-5.210946-0.178514479$ s 81 s 87 s $88 \mathrm{~s}$ atom $81-C * *-\odot 3.348737-6.0366971 .076274480 \mathrm{~s} 82 \mathrm{~s} 85 \mathrm{~s} 86 \mathrm{~s}$ atom $82-C * *-02.178081-5.677621 .996053478 \mathrm{~s} 81 \mathrm{~s} 83 \mathrm{~s} 84 \mathrm{~s}$ atom $83-\mathrm{H}^{* *}-02.417509-5.7506053 .078122182 \mathrm{~s}$ atom $84-H^{* *}-01.283396-6.3123291 .786925182 \mathrm{~s}$ atom $85-\mathrm{H}^{* *}-04.310802-5.7008771 .523627181 \mathrm{~s}$ atom $86-H^{* *}-03.413718-7.1283710 .879108181 \mathrm{~s}$ atom $87-H^{* *}-\odot 2.227371-5.717687-0.784941180 \mathrm{~s}$ atom $88-H^{* *}-03.890126-5.024695-0.83071180 \mathrm{~s}$ atom $89-H^{* *}-01.66254-3.443533-0.187429179 \mathrm{~s}$ atom $90-\mathrm{H}^{* *}-03.258668-3.1759950 .628797179 \mathrm{~s}$ 
atom $91-0 * *-0-0.177014-4.5713844 .017209377$ s 92 s $95 \mathrm{~s}$ atom $92-C * *-\odot-0.774347-5.8705054 .101181491$ s 93 s 102 s $103 \mathrm{~s}$ atom $93-C * *-\odot-1.841529-5.7545885 .213687492$ s 94 s 100 s $101 \mathrm{~s}$ atom $94-C * *-0-1.354888-4.5426966 .064338493$ s 95 s 98 s $99 \mathrm{~s}$ atom $95-C^{* *}-\odot-0.0431178-4.1138155 .374007491$ s 94 s 96 s $97 \mathrm{~s}$ atom $96-\mathrm{H}^{* *}-00.840054-4.6062835 .846068195 \mathrm{~s}$ atom $97-\mathrm{H}^{* *}-00.140697-3.021415 .349835195 \mathrm{~s}$ atom $98-\mathrm{H}^{* *}-0-1.188025-4.8046397 .130142194 \mathrm{~s}$ atom $99-H^{* *}-0-2.096958-3.7175216 .035458194 \mathrm{~s}$ atom $100-H^{* *}-0-2.842247-5.5562674 .776749193 \mathrm{~s}$ atom $101-\mathrm{H} * *-0-1.914926-6.6916725 .805211193 \mathrm{~s}$ atom $102-H^{* *}-0-1.168279-6.1163633 .093488192 \mathrm{~s}$ atom $103-\mathrm{H}^{* *}-00.00699265-6.6213484 .379309192 \mathrm{~s}$ atom $104-0 * *-0-0.10653-1.5868912 .7891314$ s 77 s 105 a atom $105-\mathrm{C} * *-00.219203-0.6625733 .6619633104$ a $106 \mathrm{~s} 115 \mathrm{~d}$ atom $106-\mathrm{N} * *-0-0.8972250 .1225984 .1350433105 \mathrm{~s} 107 \mathrm{~s} 111 \mathrm{~s}$ atom $107-\mathrm{C} * *-0-2.019189-0.6626954 .6324944106 \mathrm{~s} 108 \mathrm{~s} 109 \mathrm{~s} 110 \mathrm{~s}$ atom $108-H^{* *}-0-2.953114-0.0543454 .6364131107 \mathrm{~s}$ atom $109-\mathrm{H}^{* *}-0-1.854169-1.0301715 .68400711107 \mathrm{~s}$ atom $110-H^{* *}-0-2.161961-1.5418663 .97237111107 \mathrm{~s}$ atom $111-C * *-\odot-0.6253151 .2879424 .95354106$ s 112 s 113 s 114 s atom $112-H^{* *}-0-1.5637591 .8748595 .0792011111 \mathrm{~s}$ atom $113-H^{* *}-0-0.2423241 .0379065 .9809511111 \mathrm{~s}$ atom $114-H_{* *}-00.128141 .9333994 .4545031111 \mathrm{~s}$ atom $115-C * *-01.50365-0.4008864 .1043873105$ d 116 s $152 \mathrm{~s}$ atom 116 - C** - $02.708756-1.1345763 .5847194115$ s 117 s 150 s 151 s atom $117-C * *-03.348266-2.1679174 .5689444116$ s 118 s 129 s 149 s atom $118-C * *-03.49042-1.6905486 .0305183117$ s 119 d $120 \mathrm{~s}$ atom $119-0 * *-\odot 2.890593-2.3050336 .924481118 \mathrm{~d}$ atom $120-\mathrm{N} * *-04.283391-0.595586 \quad 6.351143118 \mathrm{~s} 121 \mathrm{~s} 125 \mathrm{~s}$ atom $121-\mathrm{C} * *-04.308855-0.1373027 .7326334120 \mathrm{~s} 122 \mathrm{~s} 123$ s $124 \mathrm{~s}$ atom $122-H^{* *}-05.353146-0.1217618 .1251221121 \mathrm{~s}$ atom $123-\mathrm{H}^{* *}-03.8921570 .8947437 .8162651121 \mathrm{~s}$ atom $124-\mathrm{H}^{* *}-03.693254-0.8347368 .3330691121 \mathrm{~s}$ atom 125 - C** - $\odot 5.0315190 .2310155 .42162744120$ s 126 s 127 s 128 s atom $126-\mathrm{H}^{* *}-\odot 6.0590930 .4084335 .8176911125 \mathrm{~s}$ atom $127-\mathrm{H}^{* *}-05.126301-0.2568514 .4364391125 \mathrm{~s}$ atom $128-\mathrm{H}^{* *}-04.5484411 .2278135 .274181125 \mathrm{~s}$ atom $129-C_{* *}^{*}-04.603701-2.8292673 .9492394117$ s 130 s 147 s 148 s atom $130-C * *-\odot 5.399772-3.796614 .8542074129 \mathrm{~s} 131 \mathrm{~s} 142 \mathrm{~s} 146 \mathrm{~s}$ atom $131-C * *-06.502555-4.4718654 .0126143130$ s 132 d $133 \mathrm{~s}$ atom $132-0 * *-06.289947-4.7735312 .8320111131 \mathrm{~d}$ atom $133-N * *-07.719121-4.7668654 .6121863131$ s 134 s 138 s atom $134-C_{* *}-08.744144-5.4287793 .8168864133$ s 135 s 136 s 137 s atom $135-\mathrm{H}^{* *}-08.35985-5.5406332 .7847121134 \mathrm{~s}$ atom $136-\mathrm{H}^{* *}-09.68269-4.8261843 .80620411134 \mathrm{~s}$ atom $137-\mathrm{H}^{* *}-08.984017-6.4354754 .2346431134 \mathrm{~s}$ atom $138-C^{* *}-\odot 8.08249-4.5385225 .9998694133$ s 139 s 140 s $141 \mathrm{~s}$ atom $139-\mathrm{H}^{* *}-07.249774-4.0918016 .5723121138 \mathrm{~s}$ atom $140-H^{* *}-08.360805-5.56 .4939151138 \mathrm{~s}$ atom $141-\mathrm{H}^{* *}-08.963813-3.8563816 .0741041138 \mathrm{~s}$ atom $142-C_{* *}-04.526101-4.8988215 .497374 \quad 4130$ s 143 s 144 s 145 s atom $143-\mathrm{H}^{* *}-04.0227-5.4981024 .7069441142 \mathrm{~s}$ atom $144-\mathrm{H}^{* *}-05.141616-5.5965246 .1087661142 \mathrm{~s}$ atom $145-\mathrm{H}^{* *}-03.754022-4.4482556 .1566491142 \mathrm{~s}$ atom $146-H^{* *}-\odot 5.849132-3.1935445 .67280611130 \mathrm{~s}$ atom $147-H^{* *}-04.2683-3.3913553 .0511421129 \mathrm{~s}$ atom $148-H^{* *}-05.308769-2.056493 .5628751129 \mathrm{~s}$ atom $149-H^{* *}-02.589542 \quad-2.9677334 .68303611117 \mathrm{~s}$ atom $150-H^{* *}-03.497857-0.4235593 .23550811116 \mathrm{~s}$ atom $151-H^{* *}-\odot 2.412768-1.7226682 .6879951116 \mathrm{~s}$ 
atom $152-\mathrm{H}^{* *}-\odot 1.6704250 .3376044 .9031531115 \mathrm{~s}$

endmol 1

\section{Structure $4 b$}

forcefield $\mathrm{mm}+$

sys $\odot \odot 1$

$\begin{array}{llllllllll}\text { view } 40 & 0.13555 & 64 & 24 & 0.6065911 & 0.0401897 & -0.7939975 & -0.05120738 & 0.9986227\end{array}$

$\begin{array}{llllllll}0.01142624 & 0.7933631 & 0.03372747 & 0.6078137 & -2.0117 & 2.0316 & -64.19\end{array}$

seed -1111

mol 1

atom $1-0 * *-\odot \odot \odot \odot 32$ s $5 \mathrm{~s} 14 \mathrm{~s}$

atom $2-C^{* *}-01.442325 \odot 041 \mathrm{~s} 3 \mathrm{~s} 12 \mathrm{~s} 13 \mathrm{~s}$

atom $3-C^{* *}-01.8415351 .476876 \odot 42 \mathrm{~s} 4 \mathrm{~s} 10 \mathrm{~s} 11 \mathrm{~s}$

atom $4-C * *-00.7390282 .093175-0.88093843 \mathrm{~s} 5 \mathrm{~s} 8 \mathrm{~s} 9 \mathrm{~s}$

atom $5-C^{* *}-0-0.5027321 .271472-0.49442941 \mathrm{~s} 4 \mathrm{~s} 6 \mathrm{~s} 7 \mathrm{~s}$

atom $6-\mathrm{H}^{* *}-0-1.1767311 .080911-1.35582515 \mathrm{~s}$

atom $7-\mathrm{H}^{* *}-0-1.0794731 .7515110 .32769715 \mathrm{~s}$

atom $8-H^{* *}-00.9791211 .949384-1.95797514 \mathrm{~s}$

atom $9-H^{* *}-00.5951653 .181049-0.70843714 \mathrm{~s}$

atom $10-\mathrm{H}^{* *}-01.795681 .8730381 .03857613 \mathrm{~s}$

atom $11-\mathrm{H}^{* *}-02.866331 .646737-0.39464613 \mathrm{~s}$

atom $12-\mathrm{H}^{* *}-01.777684-0.5529570 .90114812 \mathrm{~s}$

atom $13-\mathrm{H}^{* *}-01.814283-0.523049-0.91544812 \mathrm{~s}$

atom 14 - Li ** - $0-0.962794-1.1413621 .40459641$ s 15 s 28 s $104 \mathrm{~s}$ atom $15-0$ ** $-0-2.876201-0.4663391 .029164314$ s 16 s $19 \mathrm{~s}$

atom $16-C^{* *}-0-3.8790720 .05719571 .912877415$ s 17 s 26 s 27 s

atom $17-C^{* *}-0-5.162647-0.6990971 .55679416$ s 18 s 24 s 25 s

atom $18-C_{* *}-0-5.019131-0.8340770 .0300033417$ s 19 s 22 s $23 \mathrm{~s}$

atom $19-C^{* *}-0-3.505888-1.047219-0.149131415 \mathrm{~s} 18 \mathrm{~s} 20 \mathrm{~s} 21 \mathrm{~s}$

atom $20-\mathrm{H}^{* *}-0-3.10626-0.545406-1.056682119 \mathrm{~s}$

atom $21-\mathrm{H}^{* *}-0-3.230006-2.124856-0.18631119 \mathrm{~s}$

atom $22-\mathrm{H}^{* *}-0-5.3472650 .104113-0.47011118 \mathrm{~s}$

atom $23-\mathrm{H}^{* *}-0-5.613285-1.670553-0.394075118 \mathrm{~s}$

atom $24-\mathrm{H}^{* *}-0-5.162655-1.7015042 .039916117 \mathrm{~s}$

atom $25-\mathrm{H}^{* *}-\odot-6.083348-0.1616151 .869766117 \mathrm{~s}$

atom $26-H^{* *}-\odot-3.536383-0.09928132 .956597116 \mathrm{~s}$

atom $27-\mathrm{H}^{* *}-0-4.0002221 .157051 .747399116 \mathrm{~s}$

atom $28-0 * *-0-1.082092-3.0653031 .374249314 \mathrm{~s} 29$ a $77 \mathrm{~s}$

atom $29-\mathrm{C} * *-0-1.47935-4.1035530 .673028328$ a $30 \mathrm{~s} 39 \mathrm{~d}$

atom $30-\mathrm{N} * *-0-2.140556-5.1216571 .447039329 \mathrm{~s} 31 \mathrm{~s} 35 \mathrm{~s}$

atom $31-C^{* *}-0-3.192279-4.6406392 .327443430$ s 32 s 33 s $34 \mathrm{~s}$

atom $32-\mathrm{H}^{* *}-0-3.343061-5.3376273 .184366131 \mathrm{~s}$

atom $33-\mathrm{H}^{* *}-0-4.182657-4.5357171 .800278131 \mathrm{~s}$

atom $34-\mathrm{H}^{* *}-0-2.898606-3.6442522 .713659131 \mathrm{~s}$

atom $35-C^{* *}-0-2.466658-6.3837860 .8185674030$ s 36 s 37 s $38 \mathrm{~s}$

atom $36-\mathrm{H}^{* *}-0-2.790248-7.1091551 .599742135 \mathrm{~s}$

atom $37-\mathrm{H}^{* *}-0-3.294511-6.3111680 .0599328135 \mathrm{~s}$

atom $38-H^{* *}-0-1.571711-6.7992270 .307954135 \mathrm{~s}$

atom $39-C^{* *}-0-1.28439-4.273468-0.688041329$ d 40 s $76 \mathrm{~s}$

atom $40-\mathrm{C}^{* *}-\odot-0.548214-3.279086-1.546757439 \mathrm{~s} 41 \mathrm{~s} 74 \mathrm{~s} 75 \mathrm{~s}$

atom $41-C^{* *}-0-1.303582-2.857856-2.84024440$ s 42 s 53 s 73 s

atom $42-C^{* *}-0-1.913444-3.974881-3.715331341 \mathrm{~s} 43 \mathrm{~d} 44 \mathrm{~s}$

atom $43-0 * *-0-3.112478-3.897895-4.027723142$ d

atom $44-N^{* *}-0-1.142073-5.030372-4.176588342$ s 45 s 49 s

atom $45-C^{* *}-0-1.797284-6.099109-4.917616444$ s 46 s 47 s 48 s

atom $46-H^{* *}-0-1.379569-6.183982-5.949095145 \mathrm{~s}$

atom $47-\mathrm{H}^{* *}-0-1.6523-7.078769-4.403765145 \mathrm{~s}$

atom $48-\mathrm{H}^{* *}-0-2.877545-5.863613-4.974698145 \mathrm{~s}$

atom $49-C^{* *}-00.276975-5.232409-3.943582444$ s 50 s 51 s 52 s

atom $50-\mathrm{H}^{* *}-00.761782-5.571163-4.888457149 \mathrm{~s}$

atom $51-\mathrm{H}^{* *}-00.769355-4.29744-3.625004149 \mathrm{~s}$ 
atom $52-\mathrm{H}^{* *}-00.463834-6.009242-3.16354149 \mathrm{~s}$

atom $53-C_{* *}-0-0.505031-1.821022-3.66871441$ s 54 s 71 s $72 \mathrm{~s}$

atom $54-C * *-\odot-1.281367-1.175198-4.835394453$ s 55 s 66 s 70 s

atom $55-C^{* *}-0-2.465653-0.347326-4.297267354$ s 56 d 57 a

atom $56-0 * *-0-2.3029080 .413929-3.329904155 \mathrm{~d}$

atom $57-N^{* *}-0-3.666511-0.426917-4.965077355$ a 58 s $62 \mathrm{~s}$

atom $58-C^{* *}-0-4.7792620 .392822-4.503246457$ s 59 s 60 s 61 s

atom $59-\mathrm{H}^{* *}-0-4.3957231 .081201-3.725582158 \mathrm{~s}$

atom $60-H^{* *}-0-5.2080680 .983594-5.345218158 \mathrm{~s}$

atom $61-\mathrm{H}^{* *}-0-5.589441-0.236673-4.065552158 \mathrm{~s}$

atom $62-C * *-\odot-3.946495-1.399374-6.024351457$ s 63 s 64 s 65 s

atom $63-\mathrm{H}^{* *}-0-3.828319-2.440948-5.651421162 \mathrm{~s}$

atom $64-\mathrm{H}^{* *}-0-4.997111-1.261781-6.352038162 \mathrm{~s}$

atom $65-H^{* *}-0-3.29861-1.242839-6.916506162 \mathrm{~s}$

atom $66-C * *-0-0.371468-0.231984-5.65399454$ s 67 s 68 s 69 s

atom $67-\mathrm{H}^{* *}-0-0.008442610 .60355-5.016201166 \mathrm{~s}$

atom $68-H^{* *}-00.512452-0.781012-6.0484166 \mathrm{~s}$

atom $69-\mathrm{H}^{* *}-\odot-0.9137230 .210948-6.518739166 \mathrm{~s}$

atom $70-\mathrm{H}^{* *}-\odot-1.640544-1.979969-5.507874154 \mathrm{~s}$

atom $71-H^{* *}-0-0.193502-1.012539-2.970459153 \mathrm{~s}$

atom $72-\mathrm{H}^{* *}-00.4292-2.271173-4.077891153 \mathrm{~s}$

atom $73-H^{* *}-0-2.223034-2.342778-2.494209141 \mathrm{~s}$

atom $74-\mathrm{H}^{* *}-00.483679-3.622312-1.814712140 \mathrm{~s}$

atom $75-H^{* *}-0-0.393034-2.342611-0.965893140 \mathrm{~s}$

atom $76-\mathrm{H}^{* *}-0-1.717591-5.150859-1.192121139 \mathrm{~s}$

atom $77-\mathrm{Li} * *-\odot 0.0958254-3.0539442 .933854428 \mathrm{~s} 78 \mathrm{~s} 91 \mathrm{~s} 104 \mathrm{~s}$

atom $78-0^{* *}-\odot 1.669021-4.2843552 .471557377$ s 79 s $82 \mathrm{~s}$

atom $79-C_{* *}-02.096006-4.3499931 .095447478$ s $80 \mathrm{~s} 89 \mathrm{~s} 90 \mathrm{~s}$

atom $80-C * *-02.146977-5.8426770 .764042479$ s 81 s $87 \mathrm{~s} 88 \mathrm{~s}$

atom $81-C^{* *}-02.63304-6.437332 .098781480 \mathrm{~s} 82 \mathrm{~s} 85 \mathrm{~s} 86 \mathrm{~s}$

atom $82-C * *-\odot 1.93406-5.5474813 .140967478$ s 81 s 83 s $84 \mathrm{~s}$

atom $83-\mathrm{H}^{* *}-02.565052-5.355414 .034022182 \mathrm{~s}$

atom $84-H^{* *}-00.957093-5.9729643 .462617182 \mathrm{~s}$

atom $85-\mathrm{H}^{* *}-\odot 3.738252-6.3407772 .18546181 \mathrm{~s}$

atom $86-H^{* *}-02.376362-7.5111392 .221142181 \mathrm{~s}$

atom $87-H^{* *}-01.124477-6.2013250 .511945180 \mathrm{~s}$

atom $88-\mathrm{H}^{* *}-02.819155-6.072125-0.0905527180 \mathrm{~s}$

atom $89-\mathrm{H}^{* *}-01.360672-3.7829910 .488744179 \mathrm{~s}$

atom $90-\mathrm{H}^{* *}-03.103344-3.8753760 .995514179 \mathrm{~s}$

atom $91-0 * *-0-0.14314-3.653394 .893775377$ s 92 s $95 \mathrm{~s}$

atom $92-C * *-0-1.306998-4.1008445 .605119491$ s 93 s 102 s 103 s

atom $93-C * *-0-1.312829-3.299276 \quad 6.910414492$ s 94 s 100 s $101 \mathrm{~s}$

atom $94-C * *-00.192494-3.2221927 .223393493$ s 95 s 98 s $99 \mathrm{~s}$

atom $95-\mathrm{C} * *-00.821083-3.0842575 .825798491 \mathrm{~s} 94 \mathrm{~s} 96 \mathrm{~s} 97 \mathrm{~s}$

atom $96-\mathrm{H}^{* *}-01.782438-3.6338595 .731449195 \mathrm{~s}$

atom $97-\mathrm{H}^{* *}-00.987269-2.0221435 .537545195 \mathrm{~s}$

atom $98-H^{* *}-\odot 0.529811-4.1607057 .716745194 \mathrm{~s}$

atom $99-\mathrm{H}^{* *}-\odot 0.459277-2.3742217 .888469194 \mathrm{~s}$

atom $100-H^{* *}-\odot-1.728159-2.2822846 .733043193 \mathrm{~s}$

atom $101-H^{* *}-0-1.90846-3.7835147 .7137193 \mathrm{~s}$

atom $102-H^{* *}-0-2.194231-3.9252464 .96227192 \mathrm{~s}$

atom $103-\mathrm{H}^{* *}-0-1.235034-5.1989725 .806806192 \mathrm{~s}$

atom $104-0 * *-00.177017-1.1286652 .992253 \quad 314$ s 77 s 105 a

atom $105-\mathrm{C} * *-00.775691-0.09748883 .5451993104$ a 106 s $115 \mathrm{~d}$

atom $106-\mathrm{N} * *-0-0.1121950 .9750093 .9115673105 \mathrm{~s} 107 \mathrm{~s} 111 \mathrm{~s}$

atom $107-C_{* *}-0-1.2854220 .5721174 .6690814106$ s 108 s 109 s $110 \mathrm{~s}$

atom $108-H^{* *}-0-2.1159241 .3035614 .5345391107 \mathrm{~s}$

atom $109-\mathrm{H}^{* *}-0-1.0804240 .4947735 .7744111107 \mathrm{~s}$

atom $110-\mathrm{H} * *-0-1.612312-0.423103 \quad 4.30731811107 \mathrm{~s}$

atom $111-\mathrm{C} * *-\odot 0.4483222 .2289074 .3674724106 \mathrm{~s} 112 \mathrm{~s} 113 \mathrm{~s} 114 \mathrm{~s}$

atom $112-\mathrm{H}^{* *}-\odot-\odot .3597742 .9940134 .4193991111 \mathrm{~s}$ 


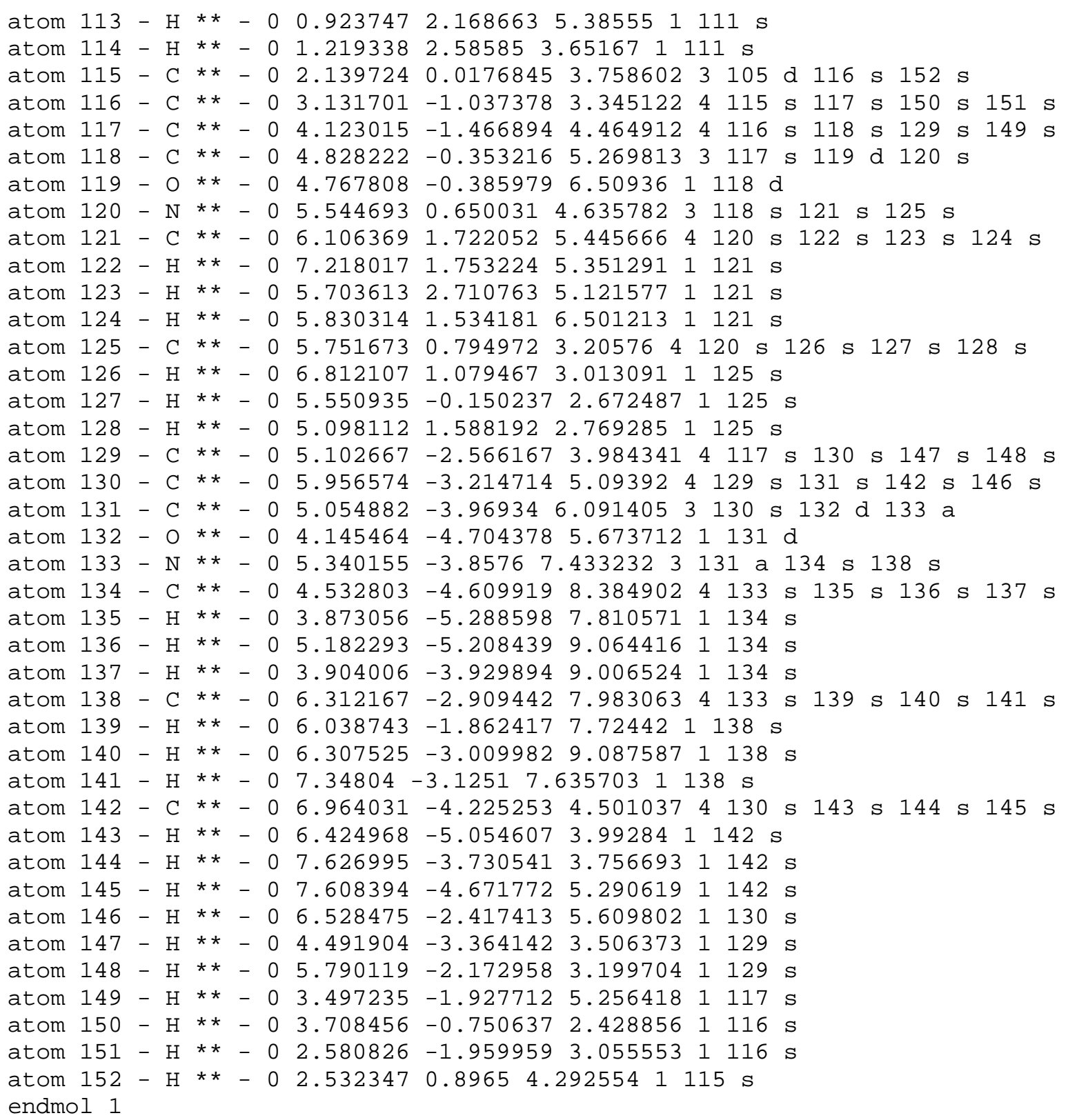

\section{Structure 5a}

forcefield $\mathrm{mm}+$

sys 0

$\begin{array}{lllllllll}\text { view } 40 & 0.095553 & 55.6 & 15.6 & -0.4114221 & -0.3861358 & -0.8256095 & -0.9109625 & 0.1447393\end{array}$ $\begin{array}{llllllll}0.3862614 & -0.02965118 & 0.9110157 & -0.4113042 & -0.22254 & -0.06932 & -55.34\end{array}$

mol 1

atom $1-C^{* *}-0-1.99476-0.53061-0.6960732 \mathrm{~s} 10 \mathrm{~d} 16 \mathrm{~s}$ atom $2-C * *-\Theta-0.89712-1.54167-0.8853941$ s 3 s 4 s 5 s atom $3-C^{* *}-0-0.86863-2.71390 .15096425 \mathrm{~s} 6 \mathrm{~s} 7 \mathrm{~s} 13 \mathrm{~s}$ atom $4-H^{* *}-0-0.93602-2.01379-1.8966912 \mathrm{~s}$ atom $5-H^{* *}-00.08139-1.01976-0.8338312 \mathrm{~s}$ atom $6-\mathrm{C} * *-\odot 0.51272-3.404650 .1038343 \mathrm{~s} 8 \mathrm{~s} 9 \mathrm{~s} 29 \mathrm{~s}$ atom $7-\mathrm{H}^{* *}-0-1.01363-2.283421 .1630613 \mathrm{~s}$ atom $8-H^{* *}-00.70859-3.69372-0.9509816 \mathrm{~s}$ atom $9-\mathrm{H}^{* *}-01.28874-2.652450 .3734516 \mathrm{~s}$ atom $10-C^{* *}-0-1.786720 .82833-0.6821531 \mathrm{~d} 11 \mathrm{~s} 12 \mathrm{~s}$ atom $11-0 * *-0-0.595651 .41249-0.95828 \quad 3 \quad 10 \mathrm{~s} 46 \mathrm{~s} 90 \mathrm{~s}$ atom $12-\mathrm{N} * *-0-2.78711 \quad 1.74876-0.27333 \quad 3 \quad 10$ s 17 s 21 s atom $13-C^{* *}-0-1.95915-3.74331-0.1936133$ s 14 d $15 \mathrm{~s}$ 


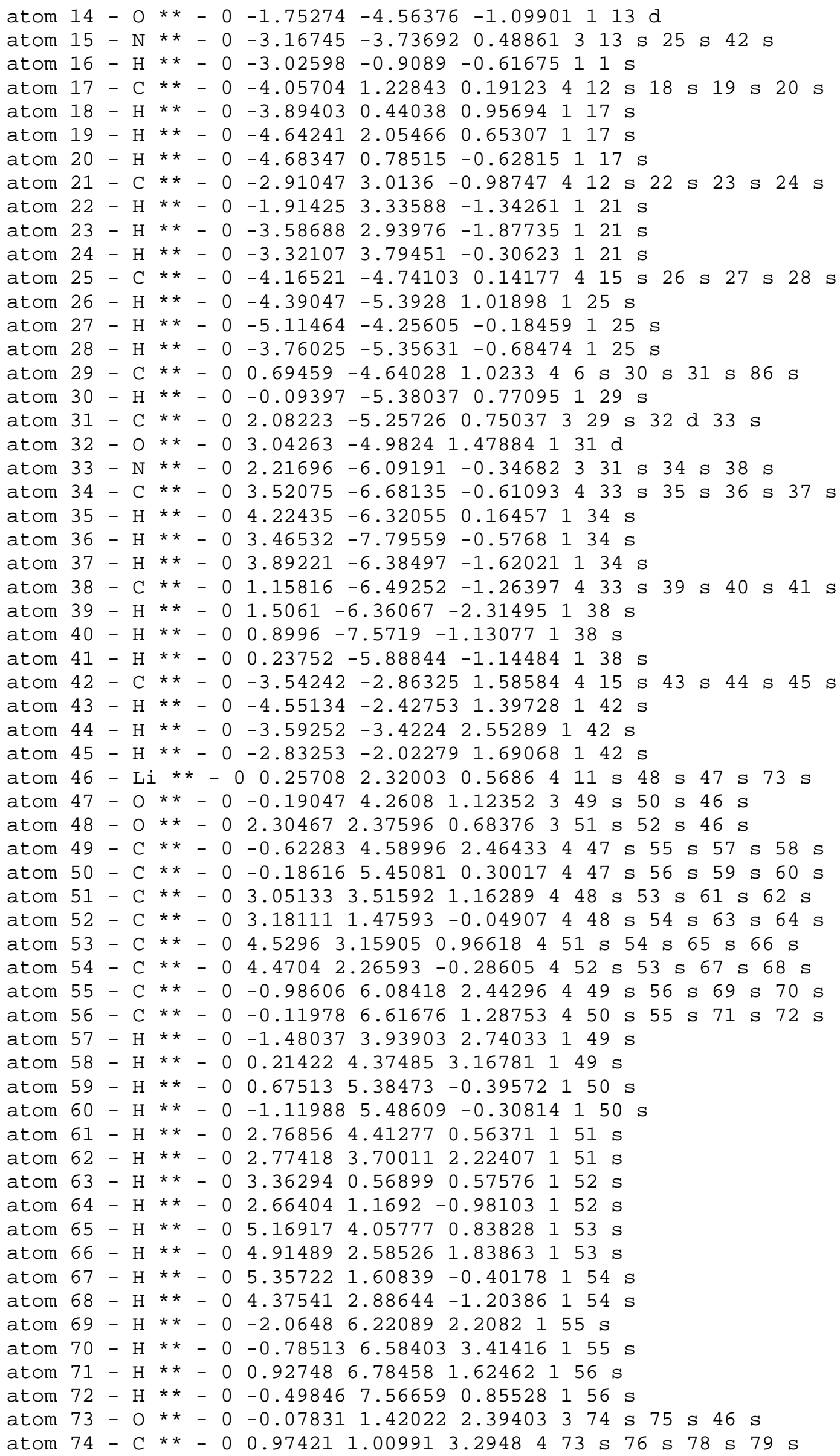


atom $75-C * *-\odot-1.34170 .839612 .80681473$ s 77 s 80 s $81 \mathrm{~s}$ atom $76-C * *-00.40629-0.173784 .08268474$ s 77 s $82 \mathrm{~s} 83 \mathrm{~s}$ atom $77-C^{* *}-0-1.082760 .203024 .17836475$ s 76 s $84 \mathrm{~s} 85 \mathrm{~s}$ atom $78-\mathrm{H}^{* *}-\odot 1.871150 .763042 .6911174 \mathrm{~s}$ atom $79-H^{* *}-\odot 1.23031 .861233 .97197174 \mathrm{~s}$ atom $80-\mathrm{H}^{* *}-\odot-2.10961 .643172 .82853175 \mathrm{~s}$ atom $81-\mathrm{H}^{* *}-\odot-1.645440 .092762 .03742175 \mathrm{~s}$ atom $82-H^{* *}-00.53549-1.120243 .51289176 \mathrm{~s}$ atom $83-H^{* *}-00.89376-0.301635 .07222176 \mathrm{~s}$ atom $84-H * *-0-1.244430 .942244 .99439177 \mathrm{~s}$ atom $85-H^{* *}-\odot-1.74372-0.667714 .37325177 \mathrm{~s}$ atom 86 - C** $-\odot 0.57624-4.296662 .51585429$ s 87 s 88 s $89 \mathrm{~s}$ atom $87-H^{* *}-\odot-0.42866-3.882272 .75227186 \mathrm{~s}$ atom $88-\mathrm{H}^{* *}-01.35446-3.562 .80893186 \mathrm{~s}$ atom $89-\mathrm{H}^{* *}-\odot 0.72731-5.198493 .14757186 \mathrm{~s}$ atom $90-\mathrm{Al} * *-\odot 0.146861 .55929-2.74788411 \mathrm{~s} 91 \mathrm{~s} 98 \mathrm{~s} 105 \mathrm{~s}$ atom $91-C^{* *}-0-1.347691 .71322-4.10222490$ s 92 s 93 s $94 \mathrm{~s}$ atom $92-C_{* *}-0-2.139190 .44257-4.47171491 \mathrm{~s} 95 \mathrm{~s} 96 \mathrm{~s} 97 \mathrm{~s}$ atom $93-\mathrm{H}^{* *}-0-0.846722 .10231-5.02631191 \mathrm{~s}$ atom $94-H^{* *}-0-2.060272 .52727-3.82202191 \mathrm{~s}$ atom $95-H^{* *}-0-2.881070 .60539-5.29289192 \mathrm{~s}$ atom $96-H^{* *}-0-1.46366-0.37658-4.81068192 \mathrm{~s}$ atom $97-H^{* *}-0-2.702090 .04009-3.59843192 \mathrm{~s}$ atom $98-C * *-01.342-0.0333-3.1557490 \mathrm{~s} 99 \mathrm{~s} 100 \mathrm{~s} 101 \mathrm{~s}$ atom $99-C^{* *}-\odot 2.23770 .18882-4.39356498$ s 102 s $103 \mathrm{~s} 104 \mathrm{~s}$ atom $100-H^{* *}-01.98325-0.35453-2.29693198 \mathrm{~s}$ atom $101-\mathrm{H}^{* *}-00.6821-0.91344-3.34804198 \mathrm{~s}$ atom $102-\mathrm{H}^{* *}-02.83935-0.71058-4.67547199 \mathrm{~s}$ atom $103-\mathrm{H}^{* *}-01.635870 .46058-5.2922199 \mathrm{~s}$ atom $104-\mathrm{H}^{* *}-02.962841 .02205-4.23942199 \mathrm{~s}$ atom $105-\mathrm{C} * *-01.209213 .30081-2.59101490$ s $106 \mathrm{~s} 107 \mathrm{~s} 108 \mathrm{~s}$ atom $106-\mathrm{C} * *-\odot 0.58524 .5434-3.259224105 \mathrm{~s} 109 \mathrm{~s} 110 \mathrm{~s} 111 \mathrm{~s}$ atom $107-\mathrm{H}^{* *}-02.213463 .12049-3.051381105 \mathrm{~s}$ atom $108-H^{* *}-01.437753 .53036-1.518341105 \mathrm{~s}$ atom $109-\mathrm{H}^{* *}-00.424524 .37792-4.348511106 \mathrm{~s}$ atom $110-H^{* *}-0-0.420224 .78685-2.840491106 \mathrm{~s}$ atom $111-H^{* *}-\odot 1.206085 .47043-3.166011106 \mathrm{~s}$ endmol 1

\section{Structure 5b}

forcefield $\mathrm{mm}+$

sys $\odot \odot 1$

$\begin{array}{llllllllll}\text { view } 40 & 0.11608 & 55.3 & 15.3 & 0.5295072 & -0.6308065 & -0.5671907 & 0.1873207 & 0.7390574 & -\end{array}$ $\begin{array}{llllllll}0.6470743 & 0.8273651 & 0.236384 & 0.5094993 & -0.81198 & 2.2304 & -53.879\end{array}$

seed -1111

$\operatorname{mol} 1$

atom $1-C^{* *}-\odot \odot \odot \odot 32 \mathrm{~s} 10 \mathrm{~d} 16 \mathrm{~s}$

atom $2-C^{* *}-01.503719 \odot 041 \mathrm{~s} 3 \mathrm{~s} 4 \mathrm{~s} 5 \mathrm{~s}$

atom $3-C^{* *}-02.2133481 .392351042 \mathrm{~s} 6 \mathrm{~s} 7 \mathrm{~s} 13 \mathrm{~s}$

atom $4-\mathrm{H}^{* *}-01.897597-0.5363510 .90076112 \mathrm{~s}$

atom $5-H^{* *}-01.864443-0.555339-0.89205912 \mathrm{~s}$

atom $6-C^{* *}-03.7421521 .18825-0.066351343$ s 8 s 9 s $29 \mathrm{~s}$

atom $7-\mathrm{H}^{* *}-01.8819281 .941418-0.9022713 \mathrm{~s}$

atom $8-H^{* *}-04.0342030 .5808680 .81898316 \mathrm{~s}$

atom $9-H^{* *}-03.9831410 .586725-0.97297916 \mathrm{~s}$

atom $10-C * * s 0-0.777448-0.608917-0.95775731$ d $11 \mathrm{~s} 12 \mathrm{~s}$

atom $11-0 * * \mathrm{~s} \odot-0.260445-1.360084-1.961051310 \mathrm{~s} 46 \mathrm{~s} 90 \mathrm{~s}$

atom $12-\mathrm{N} * *-0-2.183431-0.478472-0.990871310 \mathrm{~s} 17 \mathrm{~s} 21 \mathrm{~s}$

atom $13-C^{* *}-\odot 1.8674722 .1563261 .28930333 \mathrm{~s} 14$ d $15 \mathrm{~s}$

atom $14-0 * *-02.449014 \quad 1.8635242 .347666113 \mathrm{~d}$

atom $15-\mathrm{N} * *-00.9086953 .1499391 .252427313$ s 25 s $42 \mathrm{~s}$ 
atom $16-\mathrm{H}^{* *}-0-0.4996260 .4845270 .8536611 \mathrm{~s}$ atom $17-C_{* *}-0-2.8249340 .465853-0.102892412$ s 18 s 19 s $20 \mathrm{~s}$ atom $18-H^{* *}-\odot-2.3014671 .443336-0.144539117 \mathrm{~s}$ atom $19-\mathrm{H}^{* *}-\odot-3.8764330 .617007-0.432563117 \mathrm{~s}$ atom $20-H^{* *}-0-2.8493610 .1289850 .970135117 \mathrm{~s}$ atom $21-C * *-\odot-2.981706-1.660443-1.275493412$ s 22 s 23 s 24 s atom $22-\mathrm{H}^{* *}-0-2.423674-2.323436-1.962198121 \mathrm{~s}$ atom $23-H^{* *}-0-3.238194-2.235971-0.343355121 \mathrm{~s}$ atom $24-\mathrm{H}^{* *}-0-3.933645-1.372456-1.772992121 \mathrm{~s}$ atom $25-\mathrm{C} * *-00.6607393 .9243822 .461239415 \mathrm{~s} 26 \mathrm{~s} 27 \mathrm{~s} 28 \mathrm{~s}$ atom $26-H^{* *}-\odot 0.9019065 .0014962 .297381125 \mathrm{~s}$ atom $27-H^{* *}-0-0.4092973 .8509542 .765304125 \mathrm{~s}$ atom $28-H^{* *}-\odot 1.3023153 .5182243 .267085125 \mathrm{~s}$ atom $29-\mathrm{C} * *-04.6085542 .474803-0.10478646 \mathrm{~s} 30 \mathrm{~s} 31 \mathrm{~s} 86 \mathrm{~s}$ atom $30-\mathrm{H}^{* *}-04.3007323 .1288420 .737898129 \mathrm{~s}$ atom $31-C * *-06.0840442 .0599410 .0738546329 \mathrm{~s} 32 \mathrm{~d} 33 \mathrm{~s}$ atom $32-0 * *-\odot 6.7850111 .801722-0.910015131 \mathrm{~d}$ atom $33-\mathrm{N} * *-06.5779571 .9494741 .365599331 \mathrm{~s} 34 \mathrm{~s} 38 \mathrm{~s}$ atom $34-\mathrm{C} * *-\odot 7.9845951 .6197661 .540298433 \mathrm{~s} 35 \mathrm{~s} 36 \mathrm{~s} 37 \mathrm{~s}$ atom $35-H^{* *}-08.4282661 .4716450 .536316134 \mathrm{~s}$ atom $36-H^{* *}-08.5228452 .4425212 .068747134 \mathrm{~s}$ atom $37-H^{* *}-\odot 8.1039290 .6877772 .142151134 \mathrm{~s}$ atom $38-C * *-\odot 5.839242 .2253582 .59124433$ s 39 s 40 s $41 \mathrm{~s}$ atom $39-\mathrm{H}^{* *}-06.1368321 .483453 \quad 3.367091138 \mathrm{~s}$ atom $40-H^{* *}-06.0719153 .2413532 .996341138 \mathrm{~s}$ atom $41-\mathrm{H}^{* *}-04.7426852 .1325762 .456774138 \mathrm{~s}$ atom $42-C_{* *}-00.1754283 .5768960 .0668413415$ s 43 s $44 \mathrm{~s} 45 \mathrm{~s}$ atom $43-H^{* *}-0-0.8529593 .8759410 .367956142 \mathrm{~s}$ atom $44-\mathrm{H}^{* *}-\odot 0.6538554 .457217-0.427444142 \mathrm{~s}$ atom $45-H^{* *}-\odot 0.07557622 .756317-0.6683142 \mathrm{~s}$ atom 46 - Li ** s $00.621619-3.030121-1.442198411$ s 47 s 48 s 73 s atom $47-0^{* *}-\odot 2.650534-3.192881-1.162711346$ s 49 s $50 \mathrm{~s}$ atom $48-0 * *-00.256097-4.668172-2.616434346 \mathrm{~s} 51 \mathrm{~s} 52 \mathrm{~s}$ atom $49-\mathrm{C} * *-03.23622-3.4793330 .129188447 \mathrm{~s} 55 \mathrm{~s} 57$ s $58 \mathrm{~s}$ atom $50-C * *-03.666176-2.677139-2.064735447$ s 56 s 59 s 60 s atom $51-C * *-01.268053-5.62928-2.9922448 \mathrm{~s} 53 \mathrm{~s} 61 \mathrm{~s} 62 \mathrm{~s}$ atom $52-C * *-\odot-0.883608-4.765323-3.511296448$ s 54 s 63 s $64 \mathrm{~s}$ atom $53-C^{* *}-00.579048-6.597483-3.960153451 \mathrm{~s} 54 \mathrm{~s} 65 \mathrm{~s} 66 \mathrm{~s}$ atom $54-C^{* *}-\odot-0.428975-5.669425-4.661102452$ s 53 s 67 s 68 s atom $55-C * *-04.660869-2.9127570 .083041449$ s 56 s $69 \mathrm{~s} 70 \mathrm{~s}$ atom $56-C^{* *}-05.006177-3.023879-1.412141450$ s 55 s 71 s $72 \mathrm{~s}$ atom $57-\mathrm{H}^{* *}-02.607058-3.0142150 .917331149 \mathrm{~s}$ atom $58-H^{* *}-03.234682-4.5847340 .284075149 \mathrm{~s}$ atom $59-\mathrm{H}^{* *}-03.513472-3.141435-3.061223150 \mathrm{~s}$ atom $60-\mathrm{H}^{* *}-0 \quad 3.533047-1.576598-2.172216150 \mathrm{~s}$ atom $61-H^{* *}-02.111637-5.095111-3.487672151 \mathrm{~s}$ atom $62-H^{* *}-\odot 1.651714-6.114604-2.068968151 \mathrm{~s}$ atom $63-H^{* *}-\odot-1.738636-5.211194-2.94871152 \mathrm{~s}$ atom $64-\mathrm{H}^{* *}-0-1.163136-3.742061-3.837804152 \mathrm{~s}$ atom $65-H^{* *}-01.295137-7.079081-4.659153153 \mathrm{~s}$ atom $66-H^{* *}-00.0500075-7.402226-3.401744153 \mathrm{~s}$ atom $67-H^{* *}-0-1.273026-6.211864-5.13599154 \mathrm{~s}$ atom $68-\mathrm{H}^{* *}-\odot 0.0787285-5.063753-5.442842154 \mathrm{~s}$ atom $69-H^{* *}-04.66045-1.8462920 .396652155 \mathrm{~s}$ atom $70-\mathrm{H}^{* *}-\odot 5.361233-3.4668520 .74279155 \mathrm{~s}$ atom $71-\mathrm{H}^{* *}-05.312775-4.062168-1.670166156 \mathrm{~s}$ atom $72-\mathrm{H}^{* *}-05.818685-2.334752-1.724259156 \mathrm{~s}$ atom $73-0 * *-0-0.0794908-3.8139170 .344378346$ s 74 s $75 \mathrm{~s}$ atom $74-C^{* *}-0-0.375213-5.2247150 .477441473$ s 76 s 78 s $79 \mathrm{~s}$ atom $75-C * *-0-0.409901-3.1228821 .573701473$ s 77 s $80 \mathrm{~s} 81 \mathrm{~s}$ atom $76-C * *-0-1.0491-5.3930021 .847376474$ s 77 s 82 s 83 s 
atom $77-C^{* *}-0-0.462722-4.2146982 .644629475$ s 76 s 84 s 85 s

atom $78-\mathrm{H}^{* *}-0-1.016718-5.541167-0.371696174 \mathrm{~s}$

atom $79-\mathrm{H}^{* *}-00.582641-5.7936650 .418164174 \mathrm{~s}$

atom $80-H^{* *}-00.357163-2.3401461 .746257175 \mathrm{~s}$

atom $81-\mathrm{H}^{* *}-\odot-1.393508-2.6121451 .458576175 \mathrm{~s}$

atom $82-\mathrm{H}^{* *}-\odot-2.152507-5.2837711 .755866176 \mathrm{~s}$

atom $83-H^{* *}-\odot-0.842557-6.3843652 .303158176 \mathrm{~s}$

atom $84-\mathrm{H}^{* *}-00.560161-4.4568363 .009453177 \mathrm{~s}$

atom $85-\mathrm{H}^{* *}-0-1.08039-3.9241263 .520445177 \mathrm{~s}$

atom $86-C^{* *}-04.4529773 .257493-1.418035429$ s 87 s 88 s 89 s

atom $87-\mathrm{H}^{* *}-03.3930383 .542591-1.594716186 \mathrm{~s}$

atom $88-H^{* *}-04.8089962 .648885-2.275278186 \mathrm{~s}$

atom $89-\mathrm{H}^{* *}-05.0569254 .190928-1.39979186 \mathrm{~s}$

atom $90-A l * *-0-0.317474-0.723302-3.795131411 \mathrm{~s} 91 \mathrm{~s} 98 \mathrm{~s} 105 \mathrm{~s}$

atom $91-C * *-00.1749781 .2374-3.744717490$ s 92 s 93 s $94 \mathrm{~s}$

atom $92-C_{* *}-0-0.9698682 .237577-3.490986491$ s 95 s 96 s $97 \mathrm{~s}$

atom $93-\mathrm{H}^{* *}-00.6654921 .489586-4.71775191 \mathrm{~s}$

atom $94-\mathrm{H}^{* *}-00.9804521 .389816-2.982732191 \mathrm{~s}$

atom $95-\mathrm{H}^{* *}-0-0.636613 .305822-3.470304192 \mathrm{~s}$

atom $96-\mathrm{H}^{* *}-0-1.7549732 .166108-4.278744192 \mathrm{~s}$

atom $97-H^{* *}-\odot-1.4876462 .037718-2.524206192 \mathrm{~s}$

atom $98-C * *-0-2.161102-1.041367-4.586073490$ s 99 s 100 s 101 s

atom $99-C_{* *}-0-2.226106-0.689765-6.088155498$ s 102 s $103 \mathrm{~s} 104 \mathrm{~s}$

atom $100-H^{* *}-0-2.521429-2.093058-4.45133198 \mathrm{~s}$

atom $101-H^{* *}-0-2.905395-0.417027-4.033802198 \mathrm{~s}$

atom $102-\mathrm{H}^{* *}-0-3.241587-0.825863-6.536812199 \mathrm{~s}$

atom $103-\mathrm{H}^{* *}-0-1.9375220 .371114-6.274282199 \mathrm{~s}$

atom $104-H^{* *}-0-1.52566-1.313869-6.691357199 \mathrm{~s}$

atom $105-C * *-01.086927-1.900698-4.698299490$ s 106 s 107 s 108 s

atom $106-C * *-02.329773-1.177203-5.255544105$ s 109 s 110 s $111 \mathrm{~s}$

atom $107-\mathrm{H}^{* *}-00.580587-2.437899-5.5400251105 \mathrm{~s}$

atom $108-H^{* *}-01.414394-2.717979-4.00662711105 \mathrm{~s}$

atom $109-H^{* *}-02.047129-0.414644-6.016711106 \mathrm{~s}$

atom $110-\mathrm{H}^{* *}-02.880894-0.622555-4.4606231106 \mathrm{~s}$

atom $111-\mathrm{H}^{* *}-\odot 3.068505-1.858545-5.7478911106 \mathrm{~s}$

endmol 1

\section{Structure $5 \mathrm{c}$}

forcefield $\mathrm{mm}+$

sys $\odot \circ 1$

$\begin{array}{lllllllll}\text { view } 40 & 0.11182 & 57.4 & 17.4 & -0.2143404 & -0.7738486 & 0.5960004 & -0.4664996 & 0.6171948\end{array}$

$\begin{array}{lllllll}0.6335997 & -0.8581587 & -0.142228 & -0.4932899 & -2.1746 & 0.27165 & -59.39\end{array}$

seed -1111

mol 1

atom $1-C^{* *}-\odot \odot \odot \odot 32 \mathrm{~s} 10 \mathrm{~d} 16 \mathrm{~s}$

atom $2-C^{* *}-01.50336 \odot \odot 41 \mathrm{~s} 3 \mathrm{~s} 4 \mathrm{~s} 5 \mathrm{~s}$

atom $3-C^{* *}-02.1919881 .4070010442$ s 6 s 7 s $13 \mathrm{~s}$

atom $4-\mathrm{H}^{* *}-01.918726-0.5630020 .87001212 \mathrm{~s}$

atom $5-\mathrm{H}^{* *}-01.851288-0.542986-0.90327512 \mathrm{~s}$

atom $6-C^{* *}-03.6567181 .264154-0.46452543$ s 8 s 9 s $29 \mathrm{~s}$

atom $7-H^{* *}-01.6644082 .05053-0.73205313 \mathrm{~s}$

atom $8-\mathrm{H}^{* *}-04.1903620 .6233760 .26993416 \mathrm{~s}$

atom $9-\mathrm{H}^{* *}-03.6599510 .739834-1.44382116 \mathrm{~s}$

atom $10-C^{* *}-\odot-0.763451-0.651342-0.94065231$ d $11 \mathrm{~s} 12 \mathrm{~s}$

atom $11-0 * *-0-0.250049-1.471124-1.891928310 \mathrm{~s} 50 \mathrm{~s} 90 \mathrm{~s}$

atom $12-N^{* *}-0-2.164902-0.444762-1.055408310$ s 17 s $21 \mathrm{~s}$

atom $13-C^{* *}-02.1689222 .0046151 .41925533 \mathrm{~s} 14 \mathrm{~d} 15 \mathrm{~s}$

atom $14-0 * *-\odot 3.018911 .6496772 .244339113 \mathrm{~d}$

atom $15-N^{* *}-01.1829072 .9214811 .770339313 \mathrm{~s} 25 \mathrm{~s} 46 \mathrm{~s}$

atom $16-\mathrm{H}^{* *}-0-0.5072470 .5269650 .82307911 \mathrm{~s}$

atom $17-C^{* *}-0-2.7946960 .515108-0.171205412$ s 18 s 19 s $20 \mathrm{~s}$ 
atom $18-\mathrm{H}^{* *}-\odot-2.2425731 .479057-0.192204117 \mathrm{~s}$ atom $19-\mathrm{H}^{* *}-0-3.8375840 .6973-0.515015117 \mathrm{~s}$ atom $20-\mathrm{H}^{* *}-0-2.8450340 .1728220 .897004117 \mathrm{~s}$ atom $21-C^{* *}-\odot-3.018028-1.583241-1.370669412 \mathrm{~s} 22 \mathrm{~s} 23 \mathrm{~s} 24 \mathrm{~s}$ atom $22-H^{* *}-0-2.469291-2.278742-2.032485121 \mathrm{~s}$

atom $23-H^{* *}-0-3.331917-2.156054-0.460848121 \mathrm{~s}$ atom $24-\mathrm{H} * *-0-3.94031-1.231994-1.889825121 \mathrm{~s}$ atom $25-\mathrm{C} * *-01.2015453 .4741053 .117516415 \mathrm{~s} 26 \mathrm{~s} 27 \mathrm{~s} 28 \mathrm{~s}$ atom $26-\mathrm{H}^{* *}-01.3491994 .5806433 .092218125 \mathrm{~s}$ atom $27-H^{* *}-00.2401833 .2661013 .64326125 \mathrm{~s}$ atom $28-\mathrm{H}^{* *}-02.0366423 .0000163 .669079125 \mathrm{~s}$ atom $29-\mathrm{C} * *-\odot 4.4435822 .578584-0.60929546 \mathrm{~s} 30 \mathrm{~s} 31 \mathrm{~s} 35 \mathrm{~s}$ atom $30-C * *-05.9321662 .295497-0.920801429 \mathrm{~s} 32 \mathrm{~s} 33 \mathrm{~s} 34 \mathrm{~s}$ atom $31-H^{* *}-04.3929263 .1042330 .367997129 \mathrm{~s}$ atom $32-\mathrm{H}^{* *}-\odot 6.5341673 .228345-0.991272130 \mathrm{~s}$ atom $33-\mathrm{H}^{* *}-\odot 6.3766821 .660182-0.123917130 \mathrm{~s}$ atom $34-\mathrm{H}^{* *}-06.0314221 .753474-1.887327130 \mathrm{~s}$ atom $35-C * *-03.8593143 .459547-1.731225329 \mathrm{~s} 36 \mathrm{~d} 37 \mathrm{~s}$ atom $36-0 * *-03.2723462 .951219-2.69954135 \mathrm{~d}$ atom $37-N_{* *}-04.0479984 .831148-1.662294335 \mathrm{~s} 38 \mathrm{~s} 42 \mathrm{~s}$ atom $38-C * *-\odot 3.5896115 .659927-2.768874437$ s 39 s $40 \mathrm{~s} 41 \mathrm{~s}$ atom $39-H^{* *}-\odot 3.0973175 .002775-3.510938138 \mathrm{~s}$ atom $40-H^{* *}-02.8677946 .43128-2.411316138 \mathrm{~s}$ atom $41-H^{* *}-04.447576 \quad 6.186201-3.25044138 \mathrm{~s}$ atom $42-C^{* *}-04.7566925 .552258-0.617476437$ s 43 s 44 s $45 \mathrm{~s}$ atom $43-\mathrm{H}^{* *}-04.1293166 .393178-0.239173142 \mathrm{~s}$ atom $44-\mathrm{H}^{* *}-04.9985324 .8954450 .236801142 \mathrm{~s}$ atom $45-\mathrm{H} * *-05.7082965 .989995-1.004775142 \mathrm{~s}$ atom $46-C^{* *}-\odot 0.1556173 .4634390 .901233415$ s 47 s 48 s $49 \mathrm{~s}$ atom $47-\mathrm{H}^{* *}-\odot-0.8313533 .4369091 .419211146 \mathrm{~s}$ atom $48-H^{* *}-00.363654 .5293890 .63168146 \mathrm{~s}$ atom $49-\mathrm{H}^{* *}-00.0611622 .868706-0.025467146 \mathrm{~s}$ atom $50-\mathrm{Li} * *-0-0.207429-0.813617-3.758361411$ s 51 s 52 s 77 s atom $51-0 * *-\odot-0.8679811 .123818-3.866071350 \mathrm{~s} 53 \mathrm{~s} 54 \mathrm{~s}$ atom $52-0 * *-0-1.397367-1.626383-5.259511350 \mathrm{~s} 55 \mathrm{~s} 56 \mathrm{~s}$ atom $53-C^{* *}-0-0.3018372 .208331-3.098211451$ s 59 s 61 s 62 s atom $54-C_{* *}-\odot-2.2354751 .432504-4.214796451$ s 60 s 63 s 64 s atom $55-C_{* *}-0-2.772849-2.034947-5.279365452$ s 57 s 65 s 66 s atom $56-C * *-0-0.838956-2.074208-6.505831452$ s 58 s 67 s 68 s atom $57-C * *-\odot-2.779536-3.434626-5.92911455$ s 58 s 69 s $70 \mathrm{~s}$ atom $58-C * *-\odot-1.472537-3.455269-6.774561456$ s 57 s 71 s 72 s atom $59-C_{* *}-0-1.0961713 .440887-3.531999453$ s 60 s 73 s $74 \mathrm{~s}$ atom $60-C * *-0-2.5119342 .85264-3.681833454$ s 59 s 75 s 76 s atom $61-H^{* *}-0-0.4324451 .991037-2.011332153 \mathrm{~s}$ atom $62-\mathrm{H}^{* *}-0 \quad 0.785842 .261932-3.31372153 \mathrm{~s}$ atom $63-H^{* *}-\odot-2.3292381 .371028-5.322793154 \mathrm{~s}$ atom $64-H^{* *}-\odot-2.9081210 .673988-3.757996154 \mathrm{~s}$ atom $65-H^{* *}-\odot-3.142702-2.017779-4.23548155 \mathrm{~s}$ atom $66-H^{* *}-0-3.36778-1.306575-5.884925155 \mathrm{~s}$ atom $67-H^{* *}-0 \quad-1.105282-1.344805-7.31066156 \mathrm{~s}$ atom $68-H^{* *}-00.260944-2.092173-6.379473156 \mathrm{~s}$ atom $69-\mathrm{H} * *-0-2.755528-4.225299-5.151575157 \mathrm{~s}$ atom $70-\mathrm{H}^{* *}-0-3.691423-3.591784-6.542748157 \mathrm{~s}$ atom $71-\mathrm{H}^{* *}-0-1.66594-3.607202-7.857292158 \mathrm{~s}$ atom $72-\mathrm{H}^{* *}-\odot-0.797562-4.264085-6.429238158 \mathrm{~s}$ atom $73-H^{* *}-0-1.0414784 .268976-2.793981159 \mathrm{~s}$ atom $74-H^{* *}-\odot-0.7192323 .822859-4.507176159 \mathrm{~s}$ atom $75-H^{* *}-\odot-3.1649963 .441534-4.360001160 \mathrm{~s}$ atom $76-\mathrm{H}^{* *}-0 \quad-3.0105812 .802115-2.689368160 \mathrm{~s}$ atom $77-0 * *-01.533742-0.466101-4.786381350$ s 78 s $79 \mathrm{~s}$ atom $78-C * *-\odot 1.6735420 .559506-5.798648477$ s 80 s 82 s $83 \mathrm{~s}$ 
atom $79-C^{* *}-02.84617-0.916117-4.353818477$ s 81 s 84 s 85 s atom $80-C * *-03.1626290 .586772-6.166036478$ s 81 s 86 s $87 \mathrm{~s}$ atom $81-C * *-03.8194640 .158547-4.840992479$ s 80 s 88 s $89 \mathrm{~s}$ atom $82-\mathrm{H}^{* *}-\odot 1.009820 .307291-6.654876178 \mathrm{~s}$ atom $83-H^{* *}-\odot 1.3386141 .53162-5.373885178 \mathrm{~s}$ atom $84-H^{* *}-\odot 2.821266-1.043576-3.252448179 \mathrm{~s}$ atom $85-\mathrm{H}^{* *}-03.057648-1.910305-4.812876179 \mathrm{~s}$ atom $86-\mathrm{H}^{* *}-03.383405-0.150008-6.971006180 \mathrm{~s}$ atom $87-H^{* *}-03.4911181 .588143-6.516043180 \mathrm{~s}$ atom $88-H^{* *}-03.8403041 .012238-4.127867181 \mathrm{~s}$ atom $89-\mathrm{H}^{* *}-\odot 4.852257-0.230012-4.967013181 \mathrm{~s}$ atom $90-\mathrm{Al} * *-00.404775-3.270173-1.560483411 \mathrm{~s} 91 \mathrm{~s} 98 \mathrm{~s} 105 \mathrm{~s}$ atom $91-C_{* *}-02.164645-3.400364-0.566532490$ s 92 s 93 s $94 \mathrm{~s}$ atom $92-C_{* *}-03.510807-3.015931-1.206426491$ s 95 s 96 s $97 \mathrm{~s}$ atom $93-\mathrm{H}^{* *}-02.191661-4.496737-0.332884191 \mathrm{~s}$ atom $94-H^{* *}-02.080293-2.9169610 .436709191 \mathrm{~s}$ atom $95-H^{* *}-04.396786-3.336945-0.60414192 \mathrm{~s}$ atom $96-\mathrm{H}^{* *}-\odot 3.635107-3.465302-2.219885192 \mathrm{~s}$ atom $97-\mathrm{H}^{* *}-03.610408-1.91168-1.330474192 \mathrm{~s}$ atom $98-C * *-00.519374-3.946771-3.477863490$ s 99 s 100 s $101 \mathrm{~s}$ atom $99-C^{* *}-\odot 1.140135-5.354961-3.599587498 \mathrm{~s} 102 \mathrm{~s} 103 \mathrm{~s} 104 \mathrm{~s}$ atom $100-H^{* *}-01.093653-3.245064-4.135713198 \mathrm{~s}$ atom $101-H * *-0-0.513169-3.966491-3.90487198 \mathrm{~s}$ atom $102-\mathrm{H} * *-\odot 1.171461-5.747333-4.647454199 \mathrm{~s}$ atom $103-H^{* *}-02.187983-5.374824-3.220184199 \mathrm{~s}$ atom $104-H^{* *}-00.57586-6.104078-2.997019199 \mathrm{~s}$ atom $105-C * *-0-0.97278-4.319857-0.506859490$ s 106 s 107 s 108 s atom $106-\mathrm{C} * *-0-1.152119-3.9952290 .9900594105 \mathrm{~s} 109 \mathrm{~s} 110 \mathrm{~s} 111 \mathrm{~s}$ atom $107-\mathrm{H}^{* *}-\odot-0.619208-5.379514-0.5964581105 \mathrm{~s}$ atom $108-H^{* *}-\odot-1.970474-4.326571-1.0107991105 \mathrm{~s}$ atom $109-\mathrm{H}^{* *}-0-1.859101-4.6849681 .5161411106 \mathrm{~s}$ atom $110-H^{* *}-\odot-0.182937-4.0519471 .537011106 \mathrm{~s}$ atom $111-H * *-0-1.533523-2.9596971 .1500491106 \mathrm{~s}$ endmol 1

\section{Structure 5d}

forcefield $\mathrm{mm}+$

sys $\odot$

$\begin{array}{lllllllllll}\text { view } 40 & 0.138175515 & 0.8166912 & 0.01060568 & -0.5769774 & -0.2791647 & 0.8823148 & -\end{array}$ $\begin{array}{llllllll}0.3789296 & 0.5050569 & 0.4705402 & 0.7235395 & -0.56258 & 0.024582 & -54.686\end{array}$

mol 1

atom $1-C_{* *}-00.460040 .426221 .4591232 \mathrm{~s} 10 \mathrm{~d} 16 \mathrm{~s}$

atom $2-C_{* *}-01.062010 .010420 .1398341$ s 3 s 4 s $5 \mathrm{~s}$

atom $3-C * *-02.61393-0.019440 .1175642$ s 6 s 7 s $13 \mathrm{~s}$

atom $4-\mathrm{H}^{* *}-00.7223-1.01378-0.1594812 \mathrm{~s}$

atom $5-\mathrm{H}^{* *}-00.719130 .70059-0.6634712 \mathrm{~s}$

atom $6-C^{* *}-03.1179-0.4015-1.293143$ s 8 s 9 s $29 \mathrm{~s}$

atom $7-\mathrm{H}^{* *}-\odot 2.982491 .000290 .3351513 \mathrm{~s}$

atom $8-H^{* *}-02.64091-1.36414-1.5844916 \mathrm{~s}$

atom $9-\mathrm{H}^{* *}-\odot 2.774870 .38561-1.9991116 \mathrm{~s}$

atom $10-\mathrm{C} * *-0-0.809260 .933491 .5918931 \mathrm{~d} 11 \mathrm{~s} 12 \mathrm{~s}$

atom $11-0 * *-0-1.687390 .98050 .5589310$ s 50 s $90 \mathrm{~s}$

atom $12-\mathrm{N} * *-0-1.29331 .468522 .80348310$ s $17 \mathrm{~s} 21 \mathrm{~s}$

atom $13-C_{* *}-03.11206-1.051321 .1394533$ s 14 d $15 \mathrm{~s}$

atom $14-0 * *-02.9236-2.260710 .9286113 \mathrm{~d}$

atom $15-N^{* *}-03.773-0.62972 .28316313$ s 25 s $46 \mathrm{~s}$

atom $16-\mathrm{H}^{* *}-\odot 1.068210 .288542 .3669811 \mathrm{~s}$

atom $17-C^{* *}-\odot-0.368261 .714743 .88653412$ s $18 \mathrm{~s} 19 \mathrm{~s} 20 \mathrm{~s}$

atom $18-H^{* *}-\odot 0.532812 .23643 .50081117 \mathrm{~s}$

atom $19-H^{* *}-0-0.858152 .369124 .64204117 \mathrm{~s}$

atom $20-\mathrm{H}^{* *}-0-0.033270 .77974 .41563117 \mathrm{~s}$ 


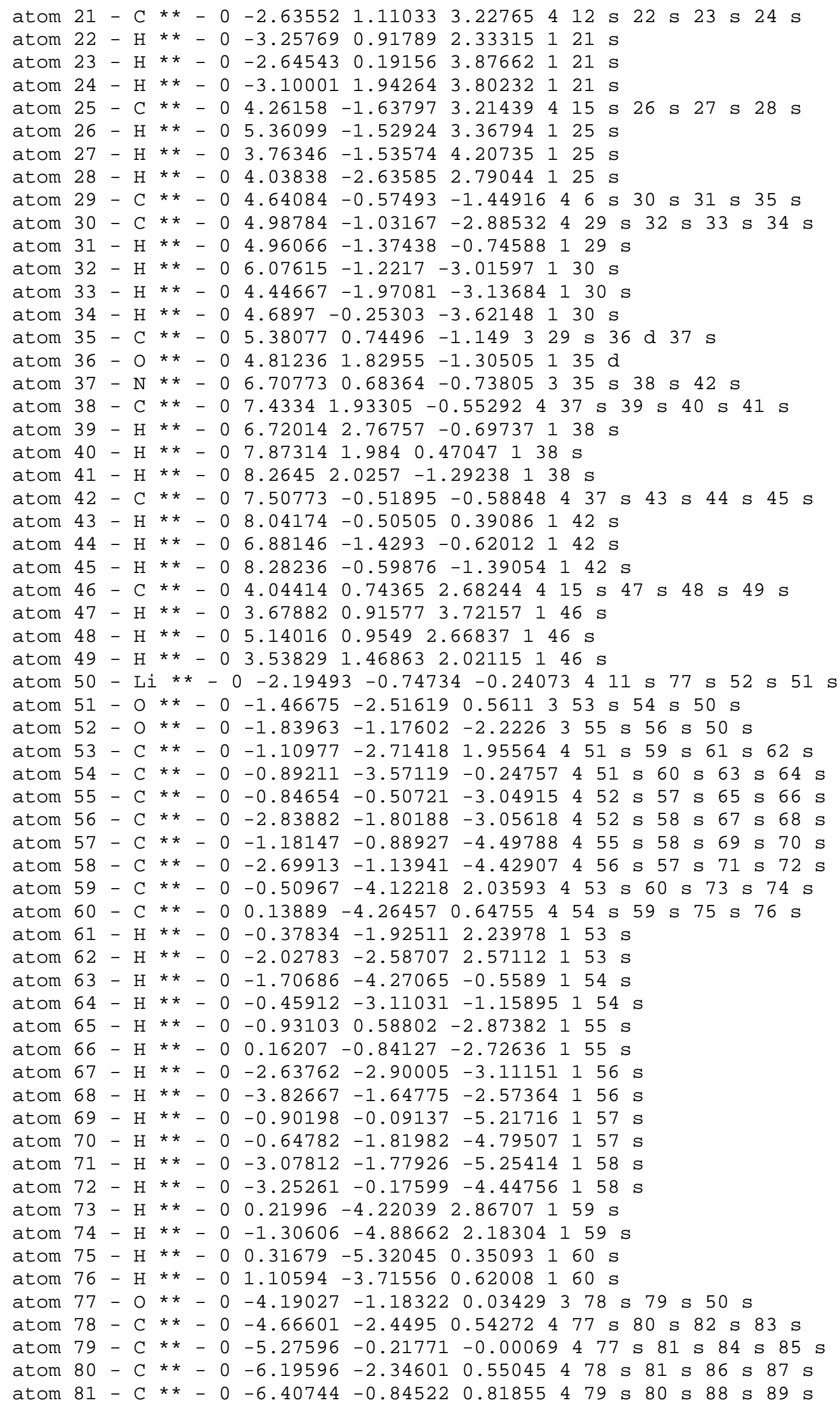




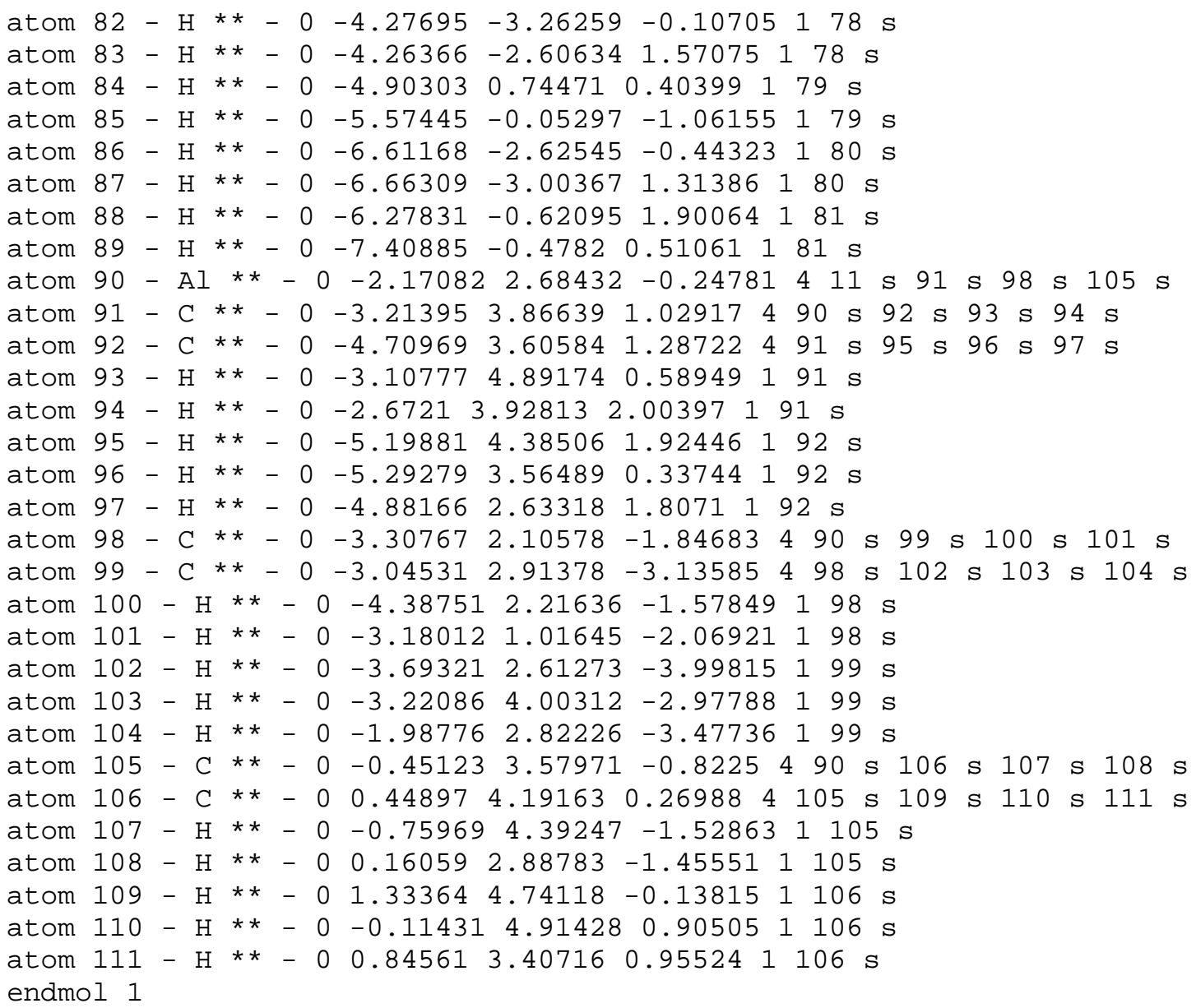

